\title{
Polytope Duality for Families of $K 3$ Surfaces and Coupling
}

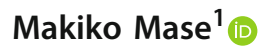 \\ Received: 12 November 2019 / Accepted: 30 May 2020 / Published online: 24 June 2020 \\ (c) The Author(s) 2020
}

\section{Abstract}

We study a relation between coupling introduced by Ebeling (Kodai Math J 29:319$336,2006)$ and the polytope duality among families of $K 3$ surfaces.

Keywords Families of $K 3$ surfaces · Coupling · Polytope duality

Mathematics Subject Classification 14J28 · 52B20

\section{Introduction}

A notion of coupling is introduced by Ebeling (2006) as a tone-down of the duality of weight systems by Kobayashi (2008). It is proved that the duality is also "polar dual", in the sense that certain rational polytopes associated to weight systems are dual. In Ebeling (2006), there is given a list of coupling pairs for 95 weight systems of simple $K 3$ hypersurface singularities classified by Yonemura (1990), and it is proved that the duality induces Saito's duality, which is a relation between the zeta functions of the Milnor fibre of the singularities. It is interesting to note that these dualities translate a famous mirror symmetry.

Instead of rational polytopes, we are interested in Batyrev's toric mirror symmetry (Batyrev 1994) for integral polytopes in this article. As a generalisation of Arnold's strange duality for unimodal singularities, Ebeling and Takahashi (2011) defined a notion of strange duality for invertible polynomials. It is studied by Mase and Ueda (2015) that the strange duality for bimodal singularities defined by invertible polynomials extends to the polytope duality among families of $K 3$ surfaces.

The polytope duality is focusing on more details in geometry of $K 3$ surfaces such as resolution of singularities, as a compactification of some singularities in three dimensional space which should affect the geometry of the surfaces while the polar duality in Ebeling (2006), Kobayashi (2008) is determined only by the weight systems

Makiko Mase

mmase@mail.uni-mannheim.de

1 Universität Mannheim, Lehrstuhl für Mathematik VI, B6, 26, 68131 Mannheim, Germany 
and thus, it is quite global. There are some profiles in coupling as is explained in Ebeling (2006) from the viewpoint of mirror symmetry, in particular, in terms of the Milnor fibres. It is expected that a study of the polar duality associated to coupling gives another explanation to coupling by Batyrev's mirror symmetry. In turn, we expect some relation can be extracted between the Milnor fibres of singularities and the geometry of associated $K 3$ surfaces.

Motivated by this, and focusing on coupling, we consider the following problem.

PROBLEM. Let $a$ and $b$ be weight systems that are coupling pair and their weighted magic square is given by polynomials $f$ and $f^{\prime}$, respectively. Determine whether or not there exist reflexive polytopes $\Delta$ and $\Delta^{\prime}$, and projectivisations $F$ and $F^{\prime}$ of $f$ and $f^{\prime}$ in the weighted projective spaces $\mathbb{P}(a)$ and $\mathbb{P}(b)$, respectively, such that they are polytope dual in the sense that they satisfy the following conditions:

$$
\Delta^{*} \simeq \Delta^{\prime}, \quad \Delta_{F} \subset \Delta \subset \Delta_{a}, \quad \Delta_{F^{\prime}} \subset \Delta^{\prime} \subset \Delta_{b}
$$

Here $\Delta_{F}$ and $\Delta_{F^{\prime}}$ are Newton polytopes of $F$ and $F^{\prime}$, respectively, and $\Delta_{a}$ and $\Delta_{b}$ are polytopes that define the weighted projective spaces $\mathbb{P}(a)$ and $\mathbb{P}(b)$. Note that the isomorphism between $\Delta^{*}$ and $\Delta^{\prime}$ is that of as integral (or lattice) polytopes.

The main theorem of the article, which is proved in Sect. 5 is stated:

Theorem 5.1 Any coupling pairs in Yonemura's list extend to the polytope dual except the following three pairs of weight systems : $(1,3,4,7 ; 15)$ (self-coupling), $(1,3,4,4 ; 12)$ (self-coupling), and $(1,1,3,5 ; 10)$ and $(3,5,11,19 ; 38)$. The complete choice of reflexive polytopes is given in Table 1 .

In Sect. 2, we recall the definitions concerning the weighted projective spaces and the strange duality. In Sect. 3, we recall the definition of coupling. In Sect. 4, we explain the polytope duality after recalling necessary notions of toric geometry.

\section{Preliminary}

A K3 surface is a compact complex 2-dimensional non-singular algebraic variety with trivial canonical bundle and irregularity zero.

Let $\left(a_{0}, \ldots, a_{n}\right)$ be a well-posed $(n+1)$-tuple of positive integers, that is, $a_{0} \leq$ $\ldots \leq a_{n}$, and any $n$-tuples out of them are coprime. Recall that the weighted projective space $\mathbb{P}(a)=\mathbb{P}\left(a_{0}, \ldots, a_{n}\right)$ with weight $a=\left(a_{0}, \ldots, a_{n}\right)$ is defined by

$$
\mathbb{P}(a)=\mathbb{P}\left(a_{0}, \ldots, a_{n}\right):=\mathbb{C}^{n+2} \backslash\{0\} / \sim
$$

where $\left(x_{0}, \ldots, x_{n}\right) \sim\left(y_{0}, \ldots, y_{n}\right)$ if there exists a non-zero complex number $\lambda$ such that

$$
\left(y_{0}, \ldots, y_{n}\right)=\left(\lambda^{a_{0}} x_{0}, \ldots, \lambda^{a_{n}} x_{n}\right)
$$

holds. We call $a_{i}$ the weight of the variable $x_{i}$, and denote it by wt $x_{i}$. 
In case $n=3$, we fix a system of variables $W, X, Y, Z$ of the weighted projective space $\mathbb{P}\left(a_{0}, a_{1}, a_{2}, a_{3}\right)$ with weights

$$
\text { wt } W=a_{0} \text {, wt } X=a_{1} \text {, wt } Y=a_{2} \text {, wt } Z=a_{3} \text {. }
$$

We say a polynomial $F$ in $\mathbb{P}\left(a_{0}, a_{1}, a_{2}, a_{3}\right)$ is an anticanonical section if $F$ is of degree $d:=a_{0}+a_{1}+a_{2}+a_{3}$. The tuple $\left(a_{0}, a_{1}, a_{2}, a_{3} ; d\right)$ is called a weight system.

By Dolgachev (1982), the anticanonical sheaf of $\mathbb{P}(a)$ is isomorphic to $\mathcal{O}_{\mathbb{P}(a)}(-d)$. All weight systems that give simple $K 3$ hypersurface singularities are classified by Yonemura (1990). Namely, if a weight $a$ is in Yonemura's list, general anticanonical sections of $\mathbb{P}(a)$ are birational to $K 3$ surfaces. Thus, one can consider families of $K 3$ surfaces.

For a polynomial $f$ in three variables, a polynomial $F$ in the weighted projective space $\mathbb{P}(a)$ is called a projectivisation of $f$ if there exists a linear form $l$ in $\mathbb{P}(a)$ such that

$$
f=\left.F\right|_{l=0}
$$

holds. In this case, the form $l$ is called a section of $f$ for $F$.

\section{Coupling}

Recall the definition of coupling for weight systems with three entries. Let $w=$ $\left(w_{1}, w_{2}, w_{3} ; d\right)$ and $w^{\prime}=\left(w_{1}^{\prime}, w_{2}^{\prime}, w_{3}^{\prime} ; h\right)$ be weight systems, with weights being well-posed.

A weighted magic square $C$ for the weight systems $w$ and $w^{\prime}$ is a square matrix of size 3 that satisfies relations

$$
C^{t}\left(w_{1} w_{2} w_{3}\right)={ }^{t}(d d d) \text { and }\left(w_{1}^{\prime} w_{2}^{\prime} w_{3}^{\prime}\right) C=(h h h) .
$$

The pair of weight systems $\left(w, w^{\prime}\right)$ is called coupled if $C$ is almost primitive, that is, if $|\operatorname{det} C|=\left(d-\sum_{i=1}^{3} w_{i}\right) h=\left(h-\sum_{i=1}^{3} w_{i}^{\prime}\right) d$ hold. The pair of weight systems $\left(w, w^{\prime}\right)$ is strongly coupled if it is coupled and the weighted magic square $C$ has entries zero in every column and row.

Thus, one can assign polynomials $f$ and $f^{\prime}$ to $C=\left(c_{i j}\right)$ in such a way that

$$
f=\sum_{i=1}^{3} x^{c_{i 1}} y^{c_{i 2}} z^{c_{i 3}}, \quad f^{\prime}=\sum_{i=1}^{3} x^{c_{1 i}} y^{c_{2 i}} z^{c_{3 i}} .
$$

In other words, there exisis weight systems $\left(a_{0}, a_{1}, a_{2}, a_{3} ; d\right)$ and $\left(b_{0}, b_{1}, b_{2}, b_{3} ; h\right)$ such that there exist $i, j \in\{0,1,2,3\}$ with properties

$$
|\operatorname{det} C|=h a_{i}=k b_{j}, \quad \text { and }{ }^{t} A_{f^{\prime}}=A_{f} \text {. }
$$


Define an anticanonical section $F$ of weight system $\left(a_{0}, a_{1}, a_{2}, a_{3} ; d\right)$ so that $l$ is the section of $f$ for $F$, where $l$ is a linear form defined by

$$
l= \begin{cases}w^{h / a_{0}} & \text { if }|\operatorname{det} C|=h a_{0}, \\ x^{h / a_{1}} & \text { if }|\operatorname{det} C|=h a_{1}, \\ y^{h / a_{2}} & \text { if }|\operatorname{det} C|=h a_{2}, \\ z^{h / a_{3}} & \text { if }|\operatorname{det} C|=h a_{3} .\end{cases}
$$

Note that the choice of variables is different from the original Ebeling's paper (Ebeling 2006). And then define a polynomial $F^{\prime}$ so that

$$
{ }^{t} A_{F^{\prime}}=A_{F}
$$

holds. Note that $F^{\prime}$ is a projectivisation of $f^{\prime}$, and an anticanonical section in the weighted projective space of weight system $\left(b_{0}, b_{1}, b_{2}, b_{3} ; h\right)$.

Ebeling (2006, Tables 2 and 3) gives (strongly) coupling pairs among weighted systems in Yonemura's list.

\section{Duality of Polytopes}

Let $M$ be a lattice of rank 3 , and $N$ be its dual lattice $\operatorname{Hom}_{\mathbb{Z}}(M, \mathbb{Z})$ that is again of rank 3. A polytope is a convex hull of finite number of points in $M \otimes \mathbb{R}$. If vertices of a polytope $\Delta$ are $v_{1}, \ldots, v_{r}$, we denot it by

$$
\Delta=\operatorname{Conv}\left\{v_{1}, \ldots, v_{r}\right\}
$$

We call a polytope integral if all the vertices of the polytope are in $M$. For a polytope $\Delta$, define the polar dual polytope $\Delta^{*}$ by

$$
\Delta^{*}:=\left\{y \in N \otimes \mathbb{R} \mid\langle y, x\rangle_{\mathbb{R}} \geq-1 \text { for all } x \in \Delta\right\}
$$

where $\langle$,$\rangle is a natural pairing N \times M \rightarrow \mathbb{Z}$, and $\langle,\rangle_{\mathbb{R}}$ is the extension to $\mathbb{R}$-coefficients. Let $\Delta$ be an integral polytope that contains the origin in its interior as the only lattice point. The polytope $\Delta$ is reflexive if the polar dual $\Delta^{*}$ is also an integral polytope.

Recall an interesting property of reflexive polytopes related to $K 3$ surfaces due to Batyrev (1994):

Theorem 4.1 (Batyrev 1994) Denote by $\mathbb{P}_{\Delta}$ the toric 3-fold associated to an integral polytope $\Delta$. The following conditions are equivalent.

(1) The polytope $\Delta$ is reflexive.

(2) General anticanonical sections of $\mathbb{P}_{\Delta}$ are birational to $K 3$ surfaces.

In particular, the weighted projective space $\mathbb{P}(a)$ with weight system $a=$ $\left(a_{0}, a_{1}, a_{2}, a_{3}\right)$ in Yonemura's list is a toric Fano 3 -fold determined by a reflexive polytope $\Delta^{(n)}$ in the $\mathbb{R}$-extension of the lattice 


$$
M_{n}:=\left\{(i, j, k, l) \in \mathbb{Z}^{4} \mid a_{0} i+a_{1} j+a_{2} k+a_{3} l=0\right\},
$$

where the weight system $a$ is assigned No. $n$ in Yonemura's list. The anticanonical sections are weighted homogeneous polynomial of degree $d:=a_{0}+a_{1}+a_{2}+a_{3}$, thus, there is a one-to-one correspondence between a lattice point $(i, j, k, l)$ in $M_{n}$ and a rational monomial $W^{i+1} X^{j+1} Y^{k+1} Z^{l+1}$. In this way, once a $\mathbb{Z}$-basis is taken for $M_{n}$, we identify lattice points in $\Delta^{(n)}$ and monomials of weighted degree $d$.

\section{Main Result}

In this section, we prove the main theorem.

Theorem 5.1 Any coupling pairs in Yonemura's list extend to the polytope dual except the following three pairs of weight systems : $(1,3,4,7 ; 15)$ (self-coupling), $(1,3,4,4 ; 12)$ (self-coupling), and $(1,1,3,5 ; 10)$ and $(3,5,11,19 ; 38)$. The complete choice of reflexive polytopes is given in Table 1.

Remark 1 In Table 1, the reflexive polytopes $\Delta$ and $\Delta^{\prime}$ are given as a set of monomials that are vertices of them. If there are more than one pairs, they are separated by a dotted line and polytopes in the same row give the polytope duality. Although they are not originally numbered, we label a number for each case treated accordingly from the top to the bottom in Tables 2 and 3 in Ebeling (2006).

Proof Take polynomials $F$ and $F^{\prime}$ that are respectively anticanonical sections of the weighted projective spaces $\mathbb{P}(a)$ and $\mathbb{P}(b)$ as in the Tables 2 and 3 in Ebeling (2006).

Recall that a pair of reflexive polytopes $\Delta$ and $\Delta^{\prime}$ is polytope dual if relations $\Delta_{F} \subset \Delta \subset \Delta_{a}, \Delta_{F^{\prime}} \subset \Delta^{\prime} \subset \Delta_{b}$, and $\Delta^{*} \simeq \Delta^{\prime}$ hold.

The strategy of the proof is that in each case, after taking a basis of the lattice $M_{n}$, we observe if the Newton polytope $\Delta_{F}$ of the polynomial $F$ is reflexive by a direct computation. If the polytope is not reflexive, then, we search a reflexive polytope $\Delta$ satisfying inclusions $\Delta_{F} \subset \Delta \subset \Delta^{(n)}$ of polytopes. The analogous observations should be made for $F^{\prime}$. Once one gets a candidate reflexive polytope $\Delta$ and $\Delta^{\prime}$, we then study whether they satisfy a relation $\Delta^{*} \simeq \Delta^{\prime}$.

The assertion is proved case by case.

\subsection{No. 1-No. 10}

We claim that the unique pair $\left(\Delta^{(14)}, \Delta^{(14)^{*}}\right)$ is polytope dual commonly for Nos. 1 to 10 . Take a basis $\left\{e_{1}^{(n)}, e_{2}^{(n)}, e_{3}^{(n)}\right\}$ of lattices $M_{n}$ for $n=14,28,45$, and 51 by

$$
\begin{array}{ll}
e_{1}^{(14)}=(-6,1,0,0), \quad e_{2}^{(14)}=(-14,0,1,0), & e_{3}^{(14)}=(-21,0,0,1), \\
e_{1}^{(28)}=(-3,1,0,0), \quad e_{2}^{(28)}=(-7,0,1,0), & e_{3}^{(28)}=(-10,0,0,1), \\
e_{1}^{(45)}=(-4,1,0,0), \quad e_{2}^{(45)}=(-9,0,1,0), & e_{3}^{(45)}=(-14,0,0,1),
\end{array}
$$




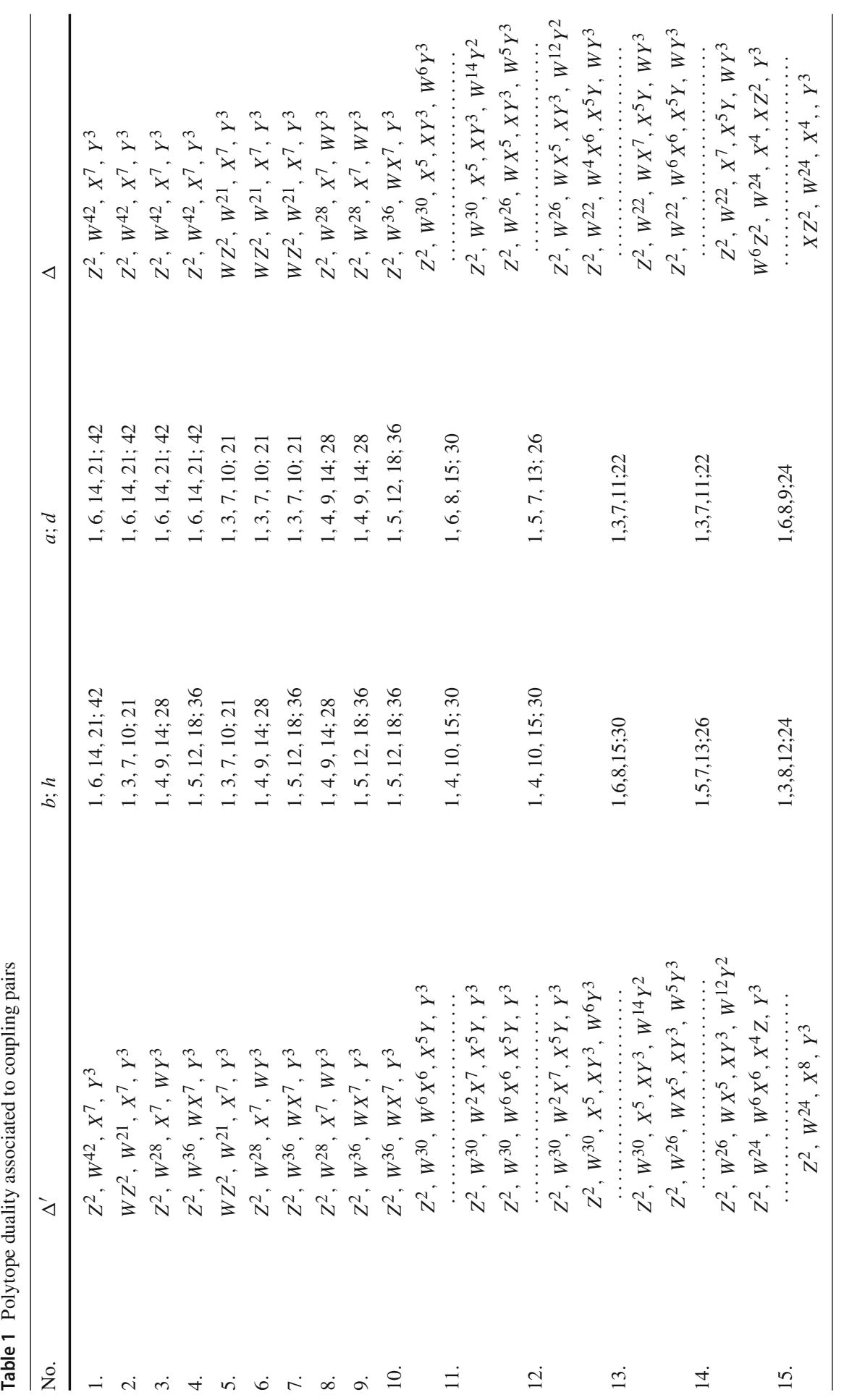

\section{Springer}




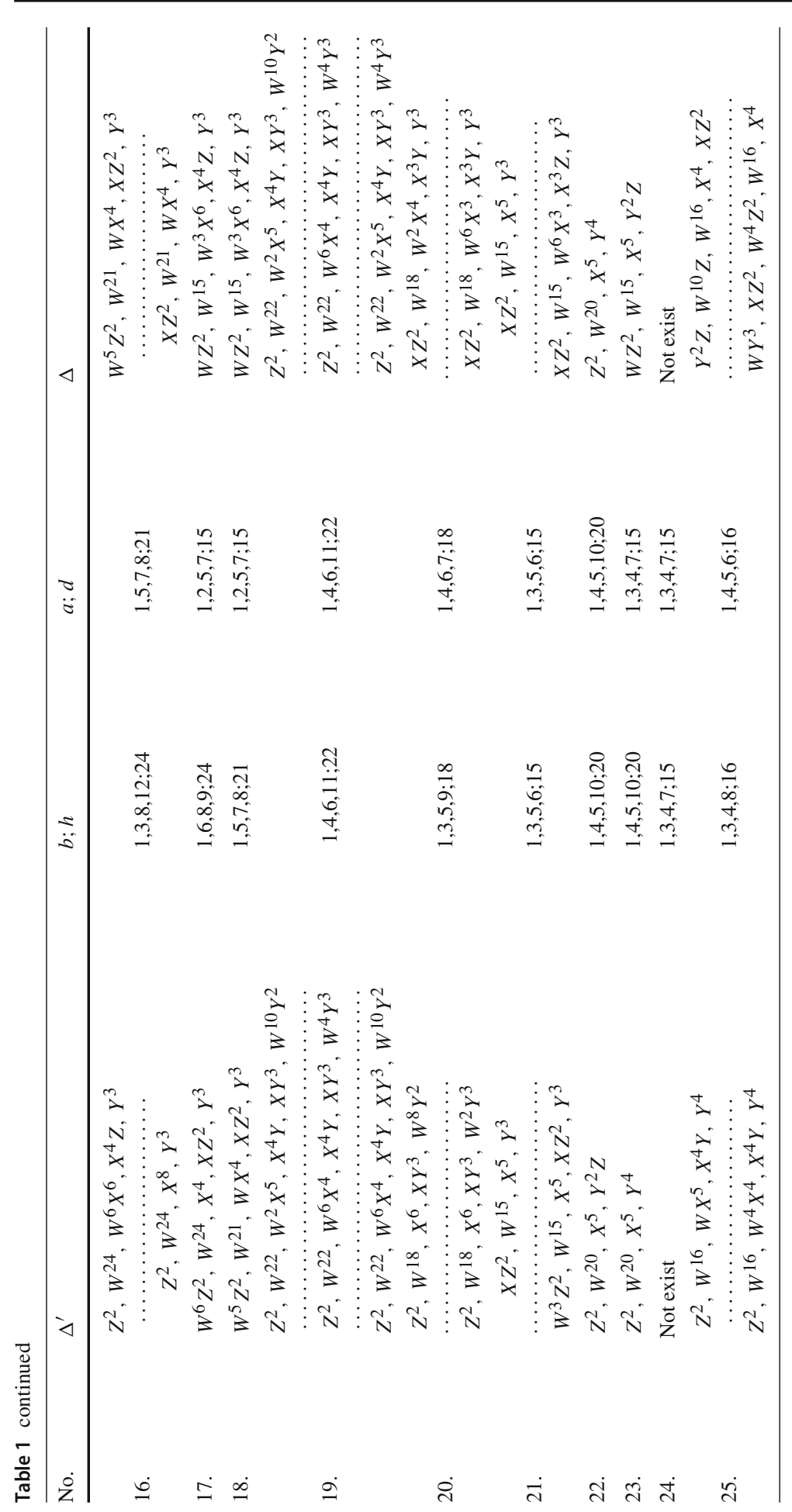




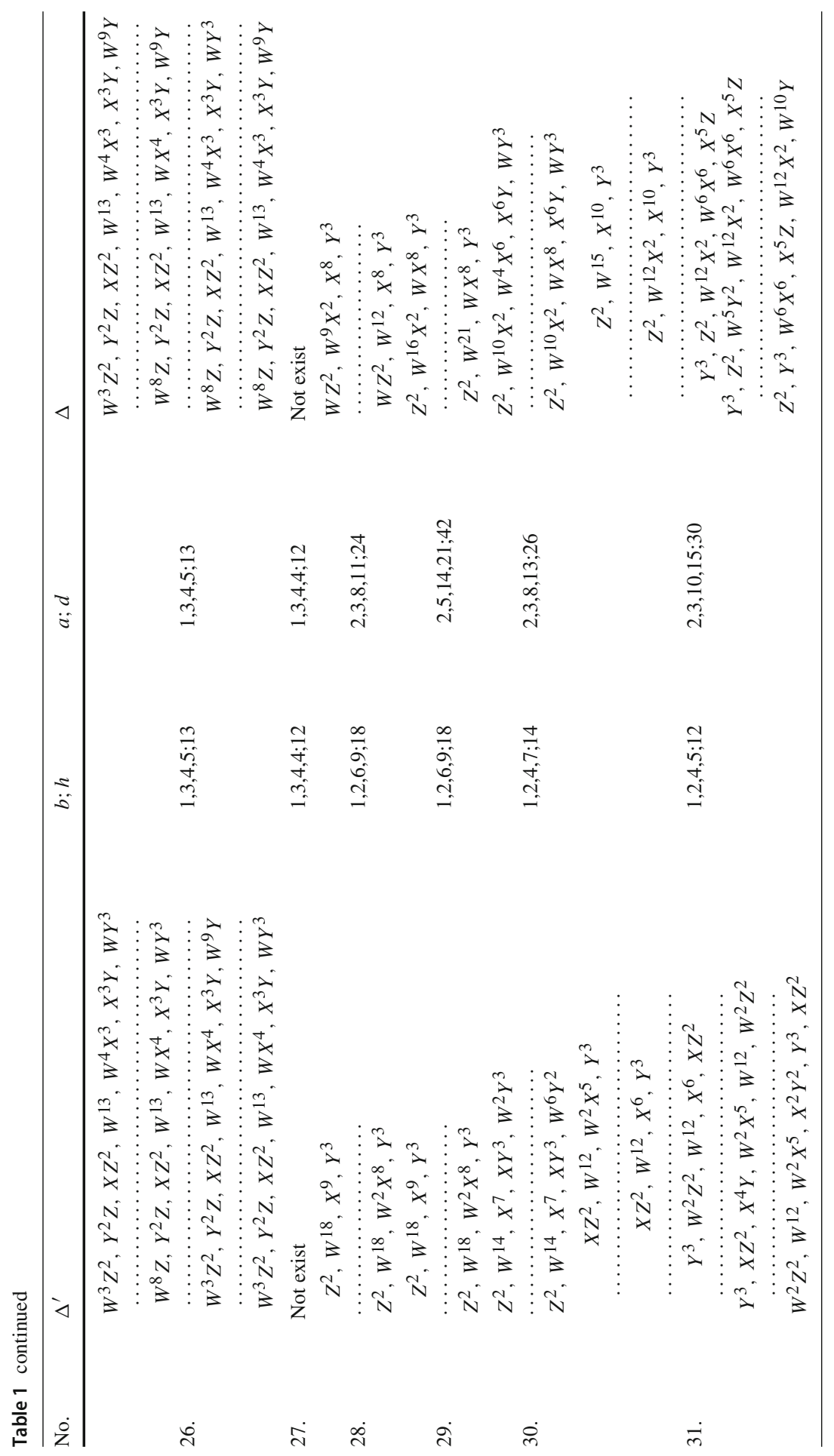




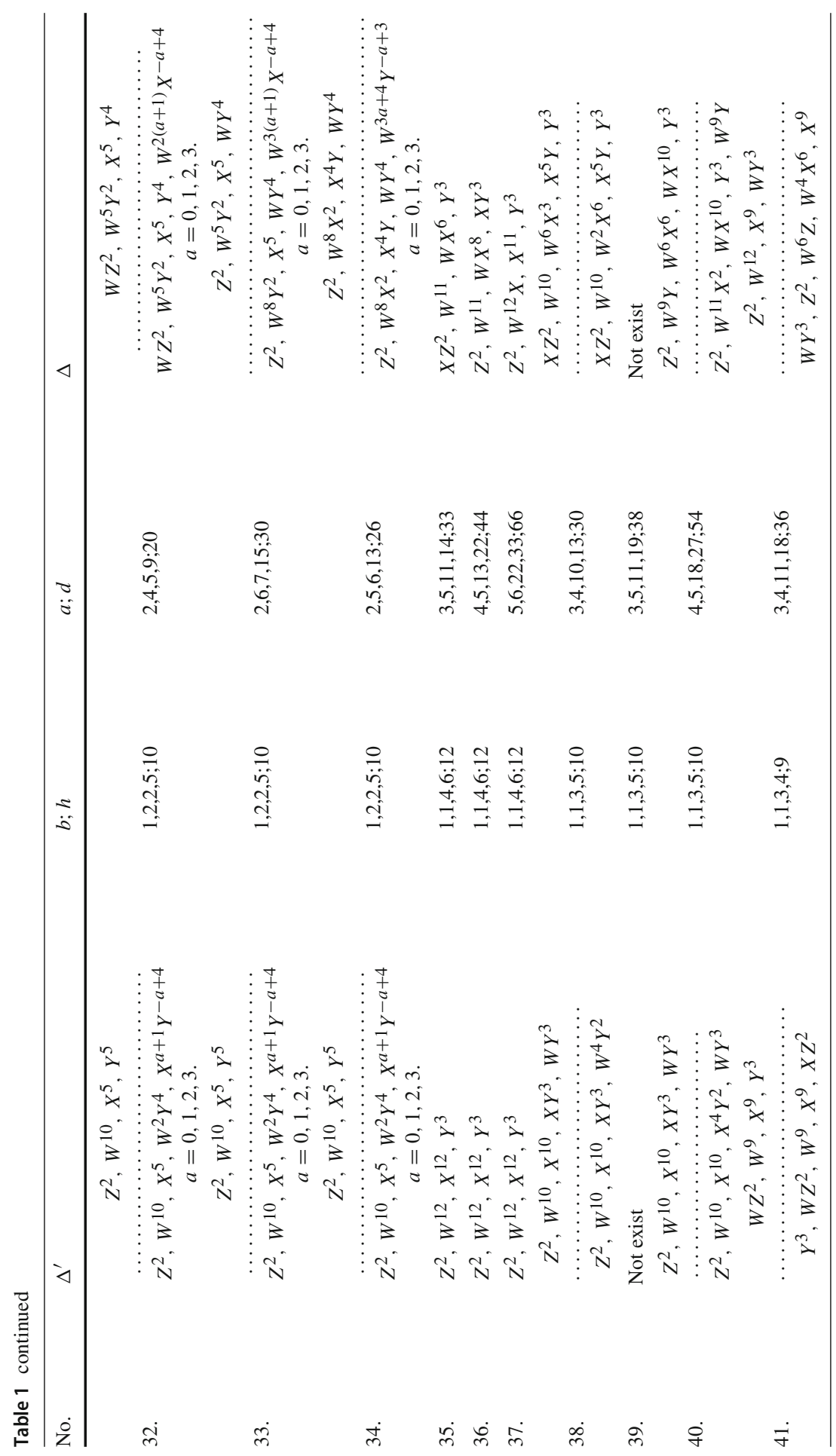




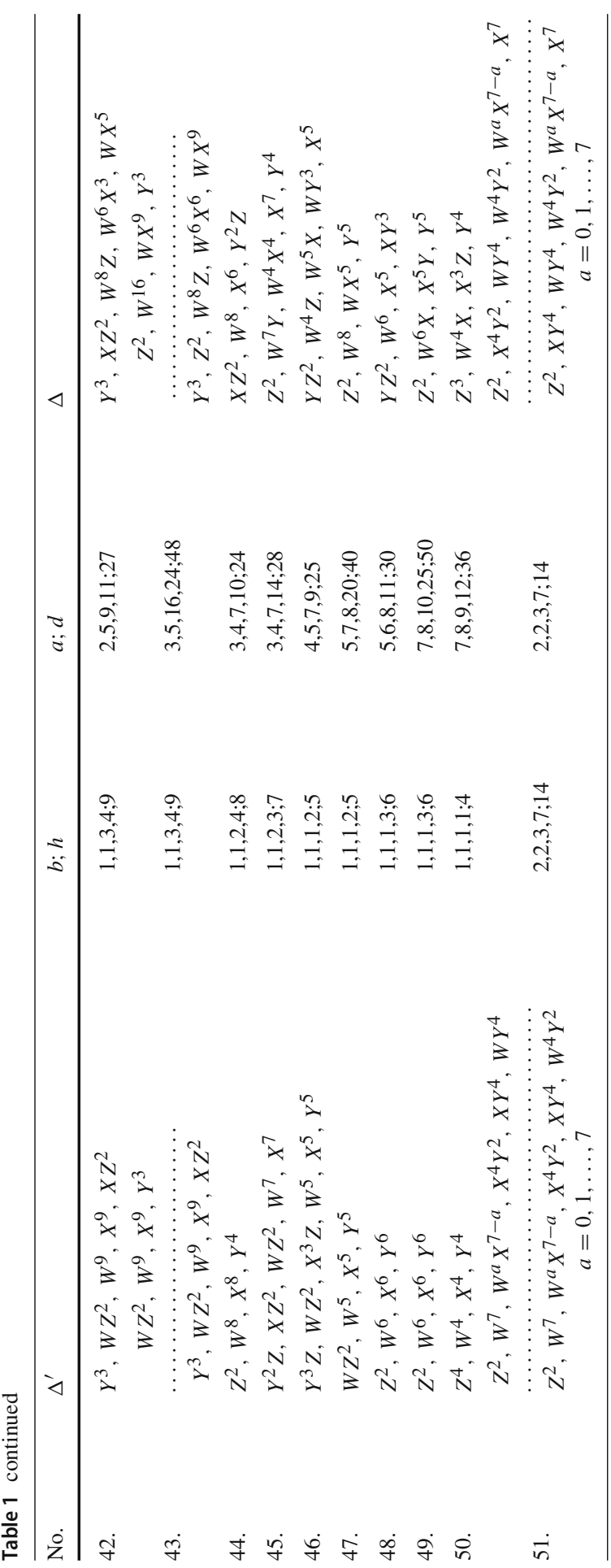




$$
e_{1}^{(51)}=(-5,1,0,0), e_{2}^{(51)}=(-12,0,1,0), \quad e_{3}^{(51)}=(-18,0,0,1) .
$$

In Kobayashi and Mase (2012), it is proved that the polytopes $\Delta^{(n)}$ for $n=$ $14,28,45$, and 51 are isomorphic to the polytope $\Delta^{(14)}$ that is the convex hull of vertices $(-1,-1,1),(-1,-1,-1),(6,-1,-1)$, and $(-1,2,-1)$ under the above choice of basis. Since the polar dual $\Delta^{(14)^{*}}$ is the convex hull of vertices $(1,0,0)$, $(0,1,0),(0,0,1)$, and $(-6,-14,-21)$, the linear map of $\mathbb{R}^{3}$ defined by a matrix $\left(\begin{array}{ccc}6 & -1 & -1 \\ -1 & 2 & -1 \\ -1 & -1 & 1\end{array}\right)$ gives an isomorphism from $\Delta^{(14)}$ to $\Delta^{(14)^{*}}$ as lattice polytopes. Therefore, a relation $\Delta^{(14)^{*}} \simeq \Delta^{(14)}$ holds.

Define 2-dimensional sections $\Gamma_{1}, \Gamma_{2}$, and $\Gamma_{3}$ of $\Delta^{(14)}$ by

$$
\begin{aligned}
& \Gamma_{1}=\operatorname{Conv}\{(-1,-1,0),(6,-1,-1),(-1,2,-1)\}, \\
& \Gamma_{2}=\operatorname{Conv}\{(-1,0,-1),(6,-1,-1),(-1,-1,1)\}, \\
& \Gamma_{3}=\operatorname{Conv}\{(0,-1,-1),(-1,-1,1),(-1,2,-1)\} .
\end{aligned}
$$

No. 1. The Newton polytope of $F=F^{\prime}$ coincides with $\Delta^{(14)}$.

No. 2. Since the Newton polytope of $F^{\prime}$, which is the convex hull of vertices $(-1,-1,1),(-1,-1,-1),(6,-1,-1)$, and $(-1,2,-1)$, coincides with $\Delta^{(28)}$, which is isomorphic to $\Delta^{(14)}$. Thus, we take $\Delta^{\prime}$ to be $\Delta^{(14)}$. Since the polar dual polytope of $\Delta^{(14)}$ is $\Delta^{(14)}$ itself, and the polynomial $F$ is an anticanonical member of the weight system $(1,6,14,21)$, the Newton polytope $\Delta_{F}$ of $F$ is a subpolytope of $\Delta$, we can take $\Delta$ to be again $\Delta^{(14)}$.

No. 3. Since the Newton polytope of $F^{\prime}$, which is the convex hull of vertices $(-1,-1,1),(-1,-1,-1),(6,-1,-1)$, and $(-1,2,-1)$, coincides with $\Delta^{(45)}$, which is isomorphic to $\Delta^{(14)}$. Thus, we take $\Delta^{\prime}$ to be $\Delta^{(14)}$. As in No.2, we can take $\Delta$ to be $\Delta^{(14)}$.

No. 4. Since the Newton polytope of $F^{\prime}$, which is the convex hull of vertices $(-1,-1,1),(-1,-1,-1),(6,-1,-1)$, and $(-1,2,-1)$, coincides with $\Delta^{(51)}$, which is isomorphic to $\Delta^{(14)}$. Thus, we take $\Delta^{\prime}$ to be $\Delta^{(14)}$. As in No.2, we can take $\Delta$ to be $\Delta^{(14)}$.

Nos. 5, 6, and 7. The Newton polytope of $F^{\prime}$, which is the convex hull of vertices $(-1,-1,0),(-1,-1,1),(6,-1,-1)$, and $(-1,2,-1)$, contains a face $\Gamma_{1}$. Since the polar dual of $\Gamma_{1}$ is a non-integral vertex $(3 / 10,7 / 10,21 / 10)$, the Newton polytope $\Delta_{F^{\prime}}$ is not reflexive. In order to construct a reflexive polytope $\Delta^{\prime}$ such that $\Delta_{F^{\prime}} \subset \Delta^{\prime}$, one may replace the face $\Gamma_{1}$ of $\Delta_{F^{\prime}}$ by some sections. In other words, fixing vertices $(-1,-1,1),(6,-1,-1)$, and $(-1,2,-1)$, we may add one or two other vertices to the polytope $\Delta_{F^{\prime}}$. This is done by a try and error construction with the following three cases.

First try-out The face $\Gamma_{1}$ is replaced by a section spanned by vertices $(-1,-1,0)$, $(6,-1,-1)$, and $(-1, a,-1)$ with $a=1,0,-1$. The polar dual of this section is a vertex $v_{1}=1 /(a+8)(a+1,7,7(a+1))$. The vertex is a lattice point if and only if $a=-1$. This means that we obtain the polytope $\Delta^{(14)}$ after the construction. Thus we may take $\Delta^{\prime}=\Delta=\Delta^{(14)}$. 
Second try-out The face $\Gamma_{1}$ is replaced by a section spanned by vertices $(-1,-1,0)$, $(-1,2,-1)$, and $(b,-1,-1)$ with $b=5,4,3,2,1,0,-1$. The polar dual of this section is a vertex $v_{2}=1 /(b+4)(3, b+1,3(b+1))$. The vertex is a lattice point if and only if $b=-1$. This means that we obtain the polytope $\Delta^{(14)}$ after the construction. Thus we may take $\Delta^{\prime}=\Delta=\Delta^{(14)}$.

Third try-out The face $\Gamma_{1}$ is replaced by a section spanned by vertices $(-1,-1,0)$, $(-1, a,-1)$, and $(b,-1,-1)$ with $a=1,0,-1$, and $b=5,4,3,2,1,0,-1$. Note that $a=-1$ if and only if $b=-1$ since this try-out means that we are constructing a polytope that contains a section which is a subset of the triangle spanned by $(-1,2,-1),(6,-1,-1)$, and $(-1,-1,-1)$ of $\Delta^{(14)}$. If $a=b=-1$, then, the resulting polytope coincides with $\Delta^{(14)}$, which is reflexive. Otherwise, the polar dual of this section is a vertex $v_{3}=1 /(a+b+2)(a+1, b+1,(a+1)(b+1))$, which is never a lattice point. Therefore, we obtain the polytope $\Delta^{(14)}$ after the construction. Thus we may take $\Delta^{\prime}=\Delta=\Delta^{(14)}$.

Nos. 8 and 9. The Newton polytope of $F^{\prime}$, which is the convex hull of vertices $(-1,0,-1),(-1,-1,1),(6,-1,-1)$, and $(-1,2,-1)$, contains a face $\Gamma_{2}$. Since the polar dual of $\Gamma_{2}$ is a non-integral vertex $(2 / 914 / 9,7 / 9)$, the Newton polytope $\Delta_{F^{\prime}}$ is not reflexive. In order to construct a reflexive polytope $\Delta^{\prime}$ such that $\Delta_{F^{\prime}} \subset \Delta^{\prime}$, one may replace the face $\Gamma_{2}$ of $\Delta_{F^{\prime}}$ by some sections. In other words, fixing vertices $(-1,-1,1),(6,-1,-1)$, and $(-1,2,-1)$, we may add one or two other vertices to the polytope $\Delta_{F^{\prime}}$. This is done by a try and error construction.

The idea to add a section two of which vertices are $(-1,-1,1)$ and $(-1,-1,0)$ is excluded by the arguments made in Nos. 5, 6, and 7. Thus we consider that we add a section spanned by vertices $(-1,-1,1),(-1,0,-1)$, and $(a,-1,-1)$ with $a=5,4,3,2,1,0,-1$. This section has the polar dual spanned by a vertex $v=$ $1 /(a+3)(2,2(a+1), a+1)$. The vertex $v$ is integral if and only if $a=-1$. Therefore, we may take $\Delta^{\prime}$ to be $\Delta^{(14)}$, and $\Delta$ to be $\Delta^{(14)}$.

No. 10. The Newton polytope of $F^{\prime}$, which is the convex hull of vertices $(0,-1,-1)$, $(-1,-1,1),(6,-1,-1)$, and $(-1,2,-1)$, contains the face $\Gamma_{3}$. Since the polar dual of $\Gamma_{3}$ is a non-integral vertex $(6 / 5,2 / 5,3 / 5)$, the Newton polytope $\Delta_{F^{\prime}}$ is not reflexive. In order to construct a reflexive polytope $\Delta^{\prime}$ such that $\Delta_{F^{\prime}} \subset \Delta^{\prime}$, one may replace the face $\Gamma_{3}$ of $\Delta_{F^{\prime}}$ by some sections. In other words, fixing vertices $(-1,-1,1)$, $(6,-1,-1)$, and $(-1,2,-1)$, we may add one or two other vertices to the polytope $\Delta_{F^{\prime}}$. This is done by a try and error construction.

The idea to add a section two of which vertices are $(-1,-1,1)$ and $(-1,-1,0)$ is excluded by the arguments made in Nos. 5, 6, and 7. Thus we consider that we add a section spanned by vertices $(-1,-1,1),(0,-1,-1)$, and $(-1, a,-1)$ with $a=1,0,-1$. This section has the polar dual spanned by a vertex $v=1 /(a+$ $3)(2(a+1), 2, a+1)$. The vertex $v$ is integral if and only if $a=-1$. Therefore, we may take $\Delta^{\prime}$ to be $\Delta^{(14)}$, and $\Delta$ to be $\Delta^{(14)}$. 


\subsection{No. $11-$ No. 14}

We claim that there exist two polytope-dual pairs for Nos. 11 to 14 . Take a basis $\left\{e_{1}^{(n)}, e_{2}^{(n)}, e_{3}^{(n)}\right\}$ of lattices $M_{n}$ for $n=38,50,77$, and 82 by

$$
\begin{aligned}
& e_{1}^{(38)}=(-6,1,0,0), \quad e_{2}^{(38)}=(-8,0,1,0), \quad e_{3}^{(38)}=(-15,0,0,1), \\
& e_{1}^{(50)}=(-4,1,0,0), \quad e_{2}^{(50)}=(-10,0,1,0), \quad e_{3}^{(50)}=(-15,0,0,1), \\
& e_{1}^{(77)}=(-5,1,0,0), \quad e_{2}^{(77)}=(-7,0,1,0), \quad e_{3}^{(77)}=(-13,0,0,1), \\
& e_{1}^{(82)}=(-3,1,0,0), \quad e_{2}^{(82)}=(-7,0,1,0), \quad e_{3}^{(82)}=(-11,0,0,1) .
\end{aligned}
$$

In Kobayashi and Mase (2012), it is proved that the polytopes $\Delta^{(38)}$ and $\Delta^{(77)}$, and $\Delta^{(50)}$, and $\Delta^{(82)}$ are respectively isomorphic to the polytopes

$$
\begin{gathered}
\Delta^{(38,77)}:=\operatorname{Conv}\left\{\begin{array}{l}
(-1,-1,1),(-1,-1,-1), \\
(4,-1,-1),(0,2,-1),(-1,2,-1)
\end{array}\right\}, \\
\Delta^{(50,82)}:=\operatorname{Conv}\left\{\begin{array}{l}
(-1,-1,1),(-1,-1,-1), \\
(6,-1,-1),(4,0,-1),(-1,2,-1)
\end{array}\right\} .
\end{gathered}
$$

Define polytopes $\Delta_{1}, \Delta_{1}^{\prime}$, and $\Delta_{2}, \Delta_{2}^{\prime}$ by

$$
\begin{aligned}
& \Delta_{1}:=\Delta^{(38,77)}, \\
& \Delta_{1}^{\prime}:=\operatorname{Conv}\left\{\begin{array}{ll}
(-1,-1,1), & (-1,-1,-1), \\
(5,-1,-1), & (4,0,-1),(-1,2,-1)
\end{array}\right\}, \\
& \Delta_{2}:=\operatorname{Conv}\left\{\begin{array}{ll}
(-1,-1,1), & (-1,-1,-1), \\
(6,-1,-1), & (4,0,-1),(-1,2,-1)
\end{array}\right\}, \\
& \Delta_{2}^{\prime}:=\Delta^{(50,82)} .
\end{aligned}
$$

Since the polar dual polytopes $\Delta_{1}^{*}$ and $\Delta_{2}^{*}$ of $\Delta_{1}$ and $\Delta_{2}$ are the convex hulls of vertices $(1,0,0),(0,1,0),(0,0,1),(0,-2,-3)$, and $(-6,-8,-15)$, respectively, $(1,0,0)$, $(0,1,0),(0,0,1),(-2,-4,-7)$, and $(-4,-10,-15)$, and the linear maps of $\mathbb{R}^{3}$ determined by matrices $A_{1}:=\left(\begin{array}{lll}1 & 1 & 2 \\ 2 & 3 & 5 \\ 3 & 4 & 8\end{array}\right)$, and $A_{2}:={ }^{t} A_{1}$ respectively give isomorphisms from $\Delta_{1}^{\prime}$ to $\Delta_{1}^{*}$ and from $\Delta_{2}^{\prime}$ to $\Delta_{2}^{*}$, the relations $\Delta_{1}^{*} \simeq \Delta_{1}^{\prime}$ and $\Delta_{2}^{*} \simeq \Delta_{2}^{\prime}$ hold.

No. 11. The Newton polytope of $F$, which is the convex hull of vertices $(-1,-1,1)$, $(-1,-1,-1),(4,-1,-1)$, and $(0,2,-1)$, is not reflexive. Indeed, $\Delta_{F}$ contains a face $\Gamma$ spanned by $(-1,-1,1),(-1,-1,-1)$, and $(0,2,-1)$ of which the polar dual is a non-integral vertex $1 / 2(3,-1,0)$. In order to construct a reflexive polytope $\Delta$ such that $\Delta_{F} \subset \Delta$, we have to replace the face $\Gamma$ by a union of some sections, which is done by add a vertex $(-1, a,-1)$ with $a=1,2$ so that there is a new face spanned by $(-1,-1,1),(0,2,-1)$, and $(-1, a,-1)$. The polar dual of this new face is a vertex $1 /(a-3)(2(a-2), 2, a+1)$. Thus, this vertex is an integral point if and only if $a=1,2$.

The Newton polytope of $F^{\prime}$, which is the convex hull of vertices $(-1,-1,1)$, $(-1,-1,-1),(4,0,-1)$, and $(-1,2,-1)$, is not reflexive. Indeed, the polytope $\Delta_{F^{\prime}}$ 
contains a face $\Gamma^{\prime}$ spanned by $(-1,-1,1),(-1,-1,-1)$, and $(4,0,-1)$ of which the polar dual is a non-integral vertex $1 / 4(-1,5,0)$. In order to construct a reflexive polytope $\Delta^{\prime}$ such that $\Delta_{F^{\prime}} \subset \Delta^{\prime}$, we have to replace the face $\Gamma^{\prime}$ by a union of some sections, which is done by add a vertex $(b,-1,-1)$ with $b=6,5,4,3,2,1,0,-1$ so that there is a new face spanned by $(-1,-1,1),(4,0,-1)$, and $(b,-1,-1)$. The polar dual of this new face is a vertex $1 /(b-7)(2,2(b-4), b+1)$. Thus, this vertex is an integral point if and only if $b=6,5$.

By the above observation, there are two polytope-dual pairs, that is, if $(a, b)=$ $(2,5)$, then, $\left(\Delta_{1}, \Delta_{1}^{\prime}\right)$, and if $(a, b)=(1,6)$, then, $\left(\Delta_{2}^{\prime}, \Delta_{2}\right)$.

No. 12. The Newton polytope of $F$, which is the convex hull of vertices $(-1,-1,1)$, $(-1,-1,-1),(4,-1,-1)$, and $(0,2,-1)$, is isomorphic to the Newton polytope of $F$ that we have already studied in No. 11 . Thus, we may take $\Delta_{1}$ or $\Delta_{2}$ as a reflexive polytope $\Delta$.

The Newton polytope of $F^{\prime}$, which is the convex hull of vertices $(4,0,-1)$, $(-1,-1,1),(0,-1,-1)$, and $(-1,2,-1)$, is not reflexive. Indeed, the polytope $\Delta_{F^{\prime}}$ contains faces $\Gamma_{1}$, which is spanned by $(-1,-1,1),(0,-1,-1)$, and $(4,0,-1)$, and $\Gamma_{2}$, which is spanned by $(-1,-1,1),(0,-1,-1)$, and $(-1,2,-1)$ of which the polar duals are vertices $v_{1}=1 / 7(-2,8,-1)$, and $v_{2}=1 / 5(6,2,3)$, respectively.

We have to replace the face $\Gamma_{1}$ by a union of two sections, in particular, we have to use a new face spanned by vertices $(-1,-1,1),(4,0,-1)$, and $(a,-1,-1)$ with $a=6,5,4,3,2,1,0,-1$. By the same argument made in No. 11 for polytope $\Delta^{\prime}$, we have to take $a=6,5$.

We have to replace $\Gamma_{2}$ by a union of two sections, in particular, we have to use a new face $\Gamma$ spanned by vertices $(-1,-1,1),(0,-1,-1)$, and $(-1, b,-1)$ with $b=-1,0,1$. The polar dual of $\Gamma$ is a vertex $1 /(b+3)(2(b+1), 2, b+1)$. Thus, this vertex is an integral point if and only if $b=-1$.

Therefore, there are two polytope-dual pairs, that is, if $(a, b)=(5,-1)$, then, $\left(\Delta_{1}, \Delta_{1}^{\prime}\right)$, and if $(a, b)=(6,-1)$, then, $\left(\Delta_{2}^{\prime}, \Delta_{2}\right)$.

No. 13. The Newton polytope of $F$, which is the convex hull of vertices $(-1,-1,1)$, $(-1,-1,-1),(4,0,-1)$, and $(-1,2,-1)$, is isomorphic to the Newton polytope of $F^{\prime}$ in No. 11 . Thus, we may take $\Delta_{1}$ or $\Delta_{2}$ as a reflexive polytope $\Delta$.

The Newton polytope of $F^{\prime}$, which is the convex hull of vertices $(0,2,-1)$, $(-1,-1,1),(-1,0,-1)$, and $(4,-1,-1)$ is not reflexive. Indeed, the polytope $\Delta_{F^{\prime}}$ contains faces $\Gamma_{1}$, which is spanned by $(-1,-1,1),(-1,0,-1)$, and $(4,-1,-1)$, and $\Gamma_{2}$, which is spanned by $(-1,-1,1),(-1,0,-1)$, and $(0,2,-1)$ of which the polar duals are vertices $v_{1}=1 / 7(2,10,5)$, and $v_{2}=1 / 3(4,-2,-1)$, respectively.

We have to replace the face $\Gamma_{1}$ by a union of two sections, in particular, we have to use a new face $\Gamma_{1}^{\prime}$ spanned by vertices $(-1,-1,1),(-1,0,-1)$, and $(a,-1,-1)$ with $a=4,3,2,1,0,-1$. The polar dual of $\Gamma_{1}^{\prime}$ is a vertex $1 /(a+3)(2,2(a+1), a+1)$. Thus, this vertex is an integral point if and only if $a=-1$.

We have to replace $\Gamma_{2}$ by a union of two sections, in particular, we have to use a new face $\Gamma_{2}^{\prime}$ spanned by vertices $(-1,-1,1),(0,2,-1)$, and $(-1, b,-1)$ with $b=1,2$. The polar dual of $\Gamma_{2}^{\prime}$ is a vertex $1 /(b-2)(2(b-2), 2, b+1)$. Thus, this vertex is an integral point if and only if $b=1,2$.

Therefore, there are two polytope-dual pairs, that is, if $(a, b)=(-1,2)$, then, $\left(\Delta_{1}^{\prime}, \Delta_{1}\right)$, and if $(a, b)=(-1,1)$, then, $\left(\Delta_{2}, \Delta_{2}^{\prime}\right)$. 
No. 14. The Newton polytope of $F$ coincides with that of $F^{\prime}$ in No. 12. Thus, one may take $\Delta_{1}$ or $\Delta_{2}$ to be $\Delta$. Besides, the Newton polytope of $F^{\prime}$ coincides with that of $F^{\prime}$ in No. 13. Thus, one may take $\Delta_{1}^{\prime}$ or $\Delta_{2}^{\prime}$ to be $\Delta^{\prime}$. Therefore, there are two polytope-dual pairs, that is, $\left(\Delta_{1}^{\prime}, \Delta_{1}\right)$ and $\left(\Delta_{2}, \Delta_{2}^{\prime}\right)$.

\subsection{No. $15-$ No. 18}

We claim that there exists a unique polytope-dual pair for Nos. 17,18 , and that two pairs for Nos. 15, 16. Take a basis $\left\{e_{1}^{(n)}, e_{2}^{(n)}, e_{3}^{(n)}\right\}$ of lattices $M_{n}$ for $n=13,20,59$, and 72 by

$$
\begin{array}{lll}
e_{1}^{(13)}=(-3,1,0,0), & e_{2}^{(13)}=(-8,0,1,0), & e_{3}^{(13)}=(-12,0,0,1), \\
e_{1}^{(20)}=(-6,1,0,0), & e_{2}^{(20)}=(-8,0,1,0), & e_{3}^{(20)}=(-9,0,0,1), \\
e_{1}^{(59)}=(-5,1,0,0), & e_{2}^{(59)}=(-7,0,1,0), & e_{3}^{(59)}=(-8,0,0,1), \\
e_{1}^{(72)}=(-2,1,0,0), & e_{2}^{(72)}=(-5,0,1,0), & e_{3}^{(72)}=(-7,0,0,1) .
\end{array}
$$

In Kobayashi and Mase (2012), it is proved that the polytopes $\Delta^{(20)}$ and $\Delta^{(59)}$ are isomorphic to the convex hull $\Delta^{(20,59)}$ of vertices $(-1,-1,1),(-1,-1,-1)$, $(3,-1,-1),(0,-1,1)$, and $(-1,2,-1)$.

Define polytopes $\Delta_{1}, \Delta_{1}^{\prime}$, and $\Delta_{2}, \Delta_{2}^{\prime}$ by

$$
\begin{aligned}
& \Delta_{1}:=\Delta^{(20,59)}, \\
& \Delta_{1}^{\prime}:=\operatorname{Conv}\left\{\begin{array}{l}
(-1,-1,1),(-1,-1,-1), \\
(5,-1,-1),(3,-1,0),(-1,2,-1)
\end{array}\right\}, \\
& \Delta_{2}:=\operatorname{Conv}\{(0,-1,1),(-1,-1,-1),(3,-1,-1),(-1,2,-1)\}, \\
& \Delta_{2}^{\prime}:=\Delta^{(13)}=\operatorname{Conv}\left\{\begin{array}{l}
(-1,-1,1),(-1,-1,-1), \\
(7,-1,-1),(-1,2,-1)
\end{array}\right\} .
\end{aligned}
$$

Since the polar dual polytopes $\Delta_{1}^{*}$ and $\Delta_{2}^{*}$ of $\Delta_{1}$ and $\Delta_{2}$ are the convex hulls of vertices $(1,0,0),(0,1,0),(0,0,1),(0,-2,-3)$, and $(-6,-8,-9)$, respectively, $(0,1,0)$, $(0,0,1),(2,0,-1)$, and $(-6,-8,-9)$, and the linear maps of $\mathbb{R}^{3}$ determined by a matrix $\left(\begin{array}{lll}1 & 1 & 1 \\ 2 & 3 & 3 \\ 3 & 4 & 5\end{array}\right)$ gives isomorphisms from $\Delta_{1}^{\prime}$ to $\Delta_{1}^{*}$ and from $\Delta_{2}^{\prime}$ to $\Delta_{2}^{*}$, the relations $\Delta_{1}^{*} \simeq \Delta_{1}^{\prime}$ and $\Delta_{2}^{*} \simeq \Delta_{2}^{\prime}$ hold

No. 15. The Newton polytope of $F$, which is the convex hull of vertices $(0,-1,1)$, $(-1,-1,-1),(3,-1,-1)$, and $(-1,2,-1)$, coincides with $\Delta_{2}$.

The Newton polytope of $F^{\prime}$, which is the convex hull of vertices $(3,-1,0)$, $(-1,-1,1),(-1,-1,-1)$, and $(-1,2,-1)$, is not reflexive. Indeed, the Newton polytope $\Delta_{F^{\prime}}$ of $F^{\prime}$ contains a face $\Gamma$ spanned by vertices $(3,-1,0),(-1,-1,-1)$, and $(-1,2,-1)$, and the polar dual of $\Gamma$ is a non-integral vertex $1 / 3(-1,0,4)$. We have to replace the face $\Gamma$ by a union of two sections, in particular, by using vertices $(3,-1,0)$ and $(-1,2,-1)$, we may choose an appropriate vertex of form $(a,-1,-1)$ with $a=7,6,5,4,3,2,1,0$, so that the resulting face is reflexive. The face $\Gamma^{\prime}$ spanned by $(3,-1,0),(-1,2,-1)$ and $(a,-1,-1)$ with $a=7,6,5,4,3,2,1,0$ has polar 
dual $v=1 /(a-8)(3, a+1,3(a-3))$. Thus, the vertex $v$ is a lattice point if and only if $a=7,5$.

Therefore, there are two polytope-dual pairs, that is, if $a=5$, then, $\left(\Delta^{(20)}=\right.$ $\left.\Delta_{1}, \Delta_{1}^{\prime}\right)$, and if $a=7$, then, $\left(\Delta_{2}, \Delta^{(13)}=\Delta_{2}^{\prime}\right)$.

No. 16. The Newton polytope of $F$ coincides with that of $F$ in No. 15, namely, with $\Delta_{2}$.

The Newton polytope of $F^{\prime}$, which is the convex hull of vertices $(-1,-1,1)$, $(0,-1,-1),(3,-1,0)$, and $(-1,2,-1)$, is not reflexive. Indeed, the Newton polytope $\Delta_{F^{\prime}}$ contains two faces $\Gamma_{1}$ spanned by $(-1,-1,1),(0,-1,-1)$, and $(-1,2,-1)$, and $\Gamma_{2}$ spanned by $(3,-1,0),(0,-1,-1)$, and $(-1,2,-1)$, and the polar dual of these faces are respectively, $\Gamma_{1}^{*}=1 / 5(6,2,3)$, and $\Gamma_{2}^{*}=1 / 8(-3,-1,9)$. We have to replace $\Gamma_{1}$ and $\Gamma_{2}$ by sums of other sections.

For $\Gamma_{1}$, we have to use a section $\Gamma_{1}^{\prime}$ spanned by $(-1,-1,1),(0,-1,-1)$, and $(-1, a,-1)$ with $a=1,0,-1$. The polar dual of $\Gamma_{1}^{\prime}$ is a vertex $v_{1}=1 /(a+3)(2(a+$ $1), 2, a+1)$. Thus, the vertex $v_{1}$ is a lattice point if and only if $a=-1$.

For $\Gamma_{2}$, we have to use a section $\Gamma_{2}^{\prime}$ spanned by $(3,-1,0),(-1,2,-1)$, and $(b,-1,-1)$ with $b=7,6,5,4,3,2,1$. The polar dual of $\Gamma_{2}^{\prime}$ is a vertex $v_{2}=$ $1 /(b-8)(3, b+1,3(b-3))$. Thus, the vertex $v_{2}$ is a lattice point if and only if $b=5,7$.

Therefore, there are two polytope-dual pairs, that is, if $b=5$, then, $\left(\Delta_{1}, \Delta_{1}^{\prime}\right)$, and if $b=7$, then, $\left(\Delta_{2}, \Delta_{2}^{\prime}\right)$.

No. 17. The Newton polytope of $F$, which is the convex hull of vertices $(-1,-1,1)$, $(-1,-1,-1),(3,-1,0)$, and $(-1,2,-1)$, coincides with that of $F^{\prime}$ in No. 15. Thus the same argument can be applied to $\Delta_{F}$, so, one may take $\Delta^{(72)}$ or a polytope with a vertex $(5,-1,-1)$ instead of a face spanned by vertices $(3,-1,0),(-1,-1,-1)$, and $(-1,2,-1)$ as $\Delta$.

The Newton polytope of $F^{\prime}$, which is the convex hull of vertices $(0,-1,1)$, $(-1,-1,0),(3,-1,-1)$, and $(-1,2,-1)$, is not reflexive. Indeed, the polytope $\Delta_{F^{\prime}}$ contains faces $\Gamma_{1}$ spanned by $(0,-1,1),(-1,-1,0)$, and $(-1,2,-1)$, and $\Gamma_{2}$ spanned by $(-1,-1,0),(3,-1,-1)$, and $(-1,2,-1)$ of which the polar duals are $\Gamma_{1}^{*}=1 / 2(3,-1,-3)$, and $\Gamma_{2}^{*}=1 / 7(3,4,12)$. We must replace these faces by sums of sections by adding other vertices to $\Delta_{F^{\prime}}$. For $\Gamma_{1}$, the only possible way to add a vertex is to adding the vertex $(-1,-1,1)$. For $\Gamma_{2}$, there are three try-outs.

First try-out Consider a section $\Gamma_{1}^{\prime}$ spanned by $(-1,-1,0),(-1,2,-1)$, and $(a,-1,-1)$ with $a=2,1,0,-1$. The polar dual of $\Gamma_{1}^{\prime}$ is a vertex $v_{1}=1 /(a+$ $4)(3, a+1,3(a+1))$. The vertex $v_{1}$ is a lattice point if and only if $a=-1$.

Second try-out Consider a section $\Gamma_{2}^{\prime}$ spanned by $(-1,-1,0),(3,-1,-1)$, and $(-1, b,-1)$ with $b=1,0,-1$. The polar dual of $\Gamma_{2}^{\prime}$ is a vertex $v_{2}=1 /(b+5)(b+$ $1,4,4(b+1))$. The vertex $v_{2}$ is a lattice point if and only if $b=-1$.

Third try-out Consider a section $\Gamma_{3}^{\prime}$ spanned by $(-1,-1,0),(a,-1,-1)$, and $(-1, b,-1)$ with $a=2,1,0,-1$ and $b=1,0,-1$. Note that $a=-1$ and $b=-1$ simultaneously occur. If $a=b=-1$, then, it is clear that $\Gamma_{3}^{\prime}$ is reflexive. Otherwise, the polar dual of $\Gamma_{3}^{\prime}$ is a vertex $v_{3}=1 /(a+b+2)(b+1, a+1,(a+1)(b+1))$, which is never a lattice point. Therefore $\Gamma_{3}^{\prime}$ is reflexive if and only if $a=b=-1$.

Therefore, the pair $\left(\Delta_{1}^{\prime}, \Delta_{1}\right)$ is polytope-dual. 
No. 18. The Newton polytope of $F$, which is the convex hull of vertices $(-1,-1,1)$, $(0,-1,-1),(3,-1,0)$, and $(-1,2,-1)$, coincides with that of $F^{\prime}$ in No. 16. Thus the same argument in No. 16 can be applied here.

The Newton polytope of $F^{\prime}$, which is the convex hull of vertices $(-1,-1,0)$, $(0,-1,1),(3,-1,-1)$, and $(-1,2,-1)$, coincides with that of $F^{\prime}$ in No. 17. Thus the same argument in No. 17 can be applied in this case.

Therefore, the pair $\left(\Delta_{1}^{\prime}, \Delta_{1}\right)$ is polytope-dual.

\subsection{No. 19}

We claim that there exist three polytope-dual pairs. Take a basis $\left\{e_{1}^{(78)}, e_{2}^{(78)}, e_{3}^{(78)}\right\}$ for a lattice $M_{78}$ by

$$
e_{1}^{(78)}=(-4,1,0,0), \quad e_{2}^{(78)}=(-6,0,1,0), \quad e_{3}^{(78)}=(-11,0,0,1) .
$$

Define polytopes $\Delta_{1}, \Delta_{2}, \Delta_{3}, \Delta_{4}$ by

$$
\begin{aligned}
& \Delta_{1}:=\Delta^{(78)}=\operatorname{Conv}\left\{\begin{array}{l}
(-1,-1,1),(-1,-1,-1),(4,-1,-1), \\
3,0,-1),(0,2,-1),(-1,2,-1)
\end{array}\right\}, \\
& \Delta_{2}:=\operatorname{Conv}\left\{\begin{array}{l}
(-1,-1,1),(-1,-1,-1),(4,-1,-1), \\
(3,0,-1),(0,2,-1),(-1,1,-1)
\end{array}\right\}, \\
& \Delta_{3}:=\operatorname{Conv}\left\{\begin{array}{l}
(-1,-1,1),(-1,-1,-1),(3,-1,-1), \\
(3,0,-1),(0,2,-1),(-1,2,-1)
\end{array}\right\}, \\
& \Delta_{4}:=\operatorname{Conv}\left\{\begin{array}{l}
(-1,-1,1),(-1,-1,-1),(3,-1,-1), \\
(3,0,-1),(0,2,-1),(-1,1,-1)
\end{array}\right\} .
\end{aligned}
$$

Since the polar dual polytopes $\Delta_{1}^{*}, \Delta_{2}^{*}$, and $\Delta_{3}^{*}$ of $\Delta_{1}, \Delta_{2}$, and $\Delta_{3}$ are the convex hulls of vertices $(1,0,0),(0,1,0),(0,0,1),(-2,-2,-5),(-4,-6,-11),(0,-2,-3)$, resp., $(1,0,0),(0,1,0),(0,0,1),(-2,-2,-5),(-4,-6,-11),(1,-1,-1)$, resp., $(1,0,0),(0,1,0),(0,0,1),(-1,0,-2),(-4,-6,-11),(0,-2,-3)$, and the linear map of $\mathbb{R}^{3}$ determined by a matrix $\left(\begin{array}{lll}1 & 1 & 2 \\ 1 & 2 & 3 \\ 2 & 3 & 6\end{array}\right)$ gives isomorphisms from $\Delta_{4}$ to $\Delta_{1}^{*}$, from $\Delta_{2}$ to $\Delta_{2}^{*}$, and from $\Delta_{3}$ to $\Delta_{3}^{*}$, the relations $\Delta_{1}^{*} \simeq \Delta_{4}, \Delta_{2}^{*} \simeq \Delta_{2}$, and $\Delta_{3}^{*} \simeq \Delta_{3}$ hold.

The Newton polytope of $F=F^{\prime}$, which is the convex hull of vertices $(-1,-1,1)$, $(-1,-1,-1),(3,0,-1)$, and $(0,2,-1)$, is not reflexive. Indeed, the polytope $\Delta_{F}$ contains faces $\Gamma_{1}$ spanned by $(-1,-1,1),(-1,-1,-1)$, and $(0,2,-1)$, and $\Gamma_{2}$ spanned by $(-1,-1,1),(-1,-1,-1)$, and $(3,0,-1)$, of which the polar duals are non-integral vertices $v_{1}=1 / 2(3,-1,0)$, and $v_{2}=1 / 3(-1,4,0)$, repsectively. For these faces, we have to replace them by sums of other sections.

For $\Gamma_{1}$, we have to use a section $\Gamma_{1}^{\prime}$ spanned by $(-1,-1,1),(0,2,-1)$, and $(-1, a,-1)$ with $a=2,1,0$. The polar dual of $\Gamma_{1}^{\prime}$ is a vertex $v_{1}=1 /(a-3)(2(a-$ $2), 2, a+1)$. The vertex $v_{1}$ is a lattice point if and only if $a=2,1$.

For $\Gamma_{2}$, we have to use a section $\Gamma_{2}^{\prime}$ spanned by $(-1,-1,1),(3,0,-1)$, and $(b,-1,-1)$ with $b=4,3,2,1,0$. The polar dual of $\Gamma_{2}^{\prime}$ is a vertex $v_{2}=1 /(b-$ $5)(2,2(b-3), b+1)$. The vertex $v_{2}$ is a lattice point if and only if $b=4,3$. 
Therefore, there are there polytope-dual pairs, that is, if $(a, b)=(2,4)$ for one side and $(a, b)=(1,3)$ for the other, then, $\left(\Delta_{1}, \Delta_{4}\right)$, if $(a, b)=(1,4)$ for both sides, then, $\left(\Delta_{2}, \Delta_{2}\right)$, if $(a, b)=(2,3)$ for both sides, then, $\left(\Delta_{3}, \Delta_{3}\right)$.

\subsection{No. 20}

We claim that there exist two polytope-dual pairs. Take a basis $\left\{e_{1}^{(n)}, e_{2}^{(n)}, e_{3}^{(n)}\right\}$ of $M_{n}$ for $n=39,60$ by

$$
\begin{aligned}
& e_{1}^{(39)}=(-3,1,0,0), \quad e_{2}^{(39)}=(-5,0,1,0), \quad e_{3}^{(39)}=(-9,0,0,1), \\
& e_{1}^{(60)}=(-4,1,0,0), \quad e_{2}^{(60)}=(-6,0,1,0), \quad e_{3}^{(60)}=(-7,0,0,1) .
\end{aligned}
$$

Define polytopes $\Delta_{1}, \Delta_{1}^{\prime}, \Delta_{2}$ and $\Delta_{2}^{\prime}$ by

$$
\begin{aligned}
& \Delta_{1}:=\operatorname{Conv}\left\{\begin{array}{l}
(0,-1,1),(-1,-1,-1), \\
(3,-1,-1),(2,0,-1),(-1,2,-1)
\end{array}\right\}, \\
& \Delta_{1}^{\prime}:=\operatorname{Conv}\left\{\begin{array}{l}
(-1,-1,1),(-1,-1,-1), \\
(5,-1,-1),(0,2,-1),(-1,1,-1)
\end{array}\right\}, \\
& \Delta_{2}:=\operatorname{Conv}\left\{\begin{array}{l}
(0,-1,1),(-1,-1,-1), \\
(2,-1,-1),(2,0,-1),(-1,2,-1)
\end{array}\right\}, \\
& \Delta_{2}^{\prime}:=\Delta^{(39)}=\operatorname{Conv}\left\{\begin{array}{l}
(-1,-1,1),(-1,-1,-1), \\
(5,-1,-1),(0,2,-1),(-1,2,-1)
\end{array}\right\} .
\end{aligned}
$$

Since the polar dual polytopes $\Delta_{1}^{*}$ and $\Delta_{2}^{*}$ of $\Delta_{1}$ and $\Delta_{2}$ are the convex hulls of vertices $(0,1,0),(0,0,1),(-2,-2,-3),(-4,-6,-7)$, and $(2,0,-1)$, respectively, $(0,1,0),(0,0,1),(-1,0,-1),(-4,-6,-7)$, and $(2,0,-1)$, and the linear map of $\mathbb{R}^{3}$ determined by a matrix $\left(\begin{array}{lll}1 & 1 & 1 \\ 1 & 2 & 2 \\ 2 & 3 & 4\end{array}\right)$ gives isomorphisms from $\Delta_{1}^{\prime}$ to $\Delta_{1}^{*}$, and from $\Delta_{2}$ to $\Delta_{2}^{*}$, the relations $\Delta_{1}^{*} \simeq \Delta_{1}^{\prime}$ and $\Delta_{2}^{*} \simeq \Delta_{2}^{\prime}$ hold.

The Newton polytope of $F$, which is the convex hull of vertices $(0,-1,1)$, $(-1,-1,-1),(2,0,-1)$, and $(-1,2,-1)$, is not reflexive. Indeed, the polytope $\Delta_{F}$ contains a face $\Gamma$ spanned by $(0,-1,1),(-1,-1,-1)$, and $(2,0,-1)$, of which the polar dual is a non-integral vertex $1 / 5(-2,6,1)$. The face $\Gamma$ has to be replaced by a sum of other sections: consider a section $\Gamma^{\prime}$ spanned by $(0,-1,1)$, $(2,0,-1)$, and $(a,-1,-1)$ with $a=3,2,1,0$. The polar dual of $\Gamma^{\prime}$ is a vertex $v^{\prime}=1 /(a-4)(2,2(a-2), a)$. Thus the vertex is a lattice point if and only if $a=2,3$.

The Newton polytope of $F^{\prime}$, which is the convex hull of vertices $(0,2,-1)$, $(-1,-1,1),(-1,-1,-1)$, and $(2,-1,0)$, is not reflexive. Indeed, the polytope $\Delta_{F^{\prime}}$ contains faces $\Gamma_{1}$ spanned by $(-1,-1,1),(-1,-1,-1)$, and $(0,2,-1)$, and $\Gamma_{2}$ spanned by $(2,-1,0),(-1,-1,-1)$, and $(0,2,-1)$, of which the polar duals are 
respectively non-lattice vertices $1 / 2(3,-1,0)$, and $1 / 7(-3,1,9)$. We have to replace these faces by sums of other sections.

For $\Gamma_{1}$, consider a section $\Gamma_{1}^{\prime}$ spanned by $(-1,-1,1),(0,2,-1)$, and $(-1, b,-1)$ with $b=2,1,0$. The polar dual of $\Gamma_{1}^{\prime}$ is a vertex $v_{1}=1 /(b-3)(2(b-2), 2, b+1)$. The vertex $v_{1}$ is a lattice point if and only if $b=1,2$.

For $\Gamma_{2}$, consider a section $\Gamma_{2}^{\prime}$ spanned by $(2,-1,0),(0,2,-1)$, and $\left(b^{\prime},-1,-1\right)$ with $b^{\prime}=5,4,3,2,1,0$. The polar dual of $\Gamma_{2}^{\prime}$ is a vertex $v_{2}=1 /\left(b^{\prime}-6\right)\left(3, b^{\prime}, 3\left(b^{\prime}-\right.\right.$ $2)$ ). The vertex $v_{2}$ is a lattice point if and only if $b^{\prime}=3,5$.

Therefore, there are two polytope-dual pairs, that is, if $\left(a, b, b^{\prime}\right)=(3,1,5)$, then, $\left(\Delta_{1}, \Delta_{1}^{\prime}\right)$, and if $\left(a, b, b^{\prime}\right)=(2,2,5)$, then, $\left(\Delta_{2}, \Delta_{2}^{\prime}\right)$.

\subsection{No. 21}

We claim that there exist two polytope-dual pairs. Take a basis $\left\{e_{1}^{(22)}, e_{2}^{(22)}, e_{3}^{(22)}\right\}$ of a lattice $M_{22}$ with by

$$
e_{1}^{(22)}=(-3,1,0,0), \quad e_{2}^{(22)}=(-5,0,1,0), \quad e_{3}^{(22)}=(-6,0,0,1)
$$

Define polytopes $\Delta_{1}, \Delta_{2}$ and $\Delta_{2}^{\prime}$ by

$$
\begin{aligned}
& \Delta_{1}:=\operatorname{Conv}\{(0,-1,1),(-1,-1,-1),(4,-1,-1),(-1,2,-1)\}, \\
& \Delta_{2}:=\operatorname{Conv}\left\{\begin{array}{l}
(0,-1,1),(-1,-1,-1), \\
(2,-1,-1),(2,-1,0),(-1,2,-1)
\end{array}\right\}, \\
& \Delta_{2}^{\prime}:=\Delta^{(22)}=\operatorname{Conv}\left\{\begin{array}{l}
(-1,-1,1),(-1,-1,-1), \\
(4,-1,-1),(0,-1,1),(-1,2,-1)
\end{array}\right\} .
\end{aligned}
$$

Since the polar dual polytopes $\Delta_{1}^{*}$ and $\Delta_{2}^{*}$ of $\Delta_{1}$ and $\Delta_{2}$ are the convex hulls of vertices $(0,1,0),(0,0,1),(-3,-5,-6)$, and $(2,0,-1)$, respectively, $(0,1,0),(0,0,1)$, $(-1,-1,0),(-3,-5,-6)$, and $(2,0,-1)$, and the linear map of $\mathbb{R}^{3}$ determined by a matrix $\left(\begin{array}{lll}1 & 1 & 1 \\ 1 & 2 & 2 \\ 1 & 2 & 3\end{array}\right)$ gives isomorphisms from $\Delta_{1}$ to $\Delta_{1}^{*}$, and from $\Delta_{2}^{\prime}$ to $\Delta_{2}^{*}$, the relations $\Delta_{1}^{*} \simeq \Delta_{1}$ and $\Delta_{2}^{*} \simeq \Delta_{2}^{\prime}$ hold.

The Newton polytope of $F=F^{\prime}$, which is the convex hull of vertices $(0,-1,1)$, $(-1,-1,-1),(2,-1,0)$, and $(-1,2,-1)$, is not reflexive. Indeed, the polytope $\Delta_{F}$ contains a face $\Gamma$ spannd by $(2,-1,0),(-1,-1,-1)$, and $(-1,2,-1)$ of which the polar dual is a non-integral vertex $1 / 2(-1,0,3)$. We may replace the face $\Gamma$ by a union of other sections. Consider a section $\Gamma^{\prime}$ spanned by $(2,-1,0),(-1,2,-1)$, and $(a,-1,-1)$ with $a=4,3,2,1,0$. The polar dual of $\Gamma^{\prime}$ is a vertex $v=1 /(a-$ $5)(3, a+1,3(a-2))$. The vertex $v$ is a lattice point if and only if $a=2,4$.

Therefore, there are two polytope-dual pairs, that is, if $a=4$, then, $\left(\Delta_{1}, \Delta_{1}\right)$, and if $a=2$ for $\Delta$, and $a=4$ for $\Delta^{\prime}$, then, $\left(\Delta_{2}, \Delta_{2}^{\prime}\right)$. 


\subsection{No. 22-No. 24}

We claim that there exist a unique polytope-dual pair for Nos. 22 and 23, and that none for No. 24. Take a basis $\left\{e_{1}^{(n)}, e_{2}^{(n)}, e_{3}^{(n)}\right\}$ of lattices $M_{n}$ for $n=9$ and 71 by

$$
\begin{gathered}
e_{1}^{(9)}=(-4,1,0,0), \quad e_{2}^{(9)}=(-5,0,1,0), \quad e_{3}^{(9)}=(-10,0,0,1), \\
e_{1}^{(71)}=(-3,1,0,0), \quad e_{2}^{(71)}=(-4,0,1,0), \quad e_{3}^{(71)}=(-7,0,0,1) .
\end{gathered}
$$

Define polytopes $\Delta_{1}$ and $\Delta_{2}$ by

$$
\begin{aligned}
& \Delta_{1}:=\Delta^{(9)}=\operatorname{Conv}\left\{\begin{array}{l}
(-1,-1,1),(-1,-1,-1), \\
(4,-1,-1),(-1,3,-1)
\end{array}\right\}, \\
& \Delta_{2}:=\operatorname{Conv}\{(-1,-1,1),(-1,-1,-1),(4,-1,-1),(-1,1,0)\} .
\end{aligned}
$$

Since the polar dual polytope $\Delta_{1}^{*}$ of $\Delta_{1}$ is the convex hull of vertices $(1,0,0),(0,1,0)$, $(0,0,1)$, and $(-4,-5,-10)$, and the linear maps of $\mathbb{R}^{3}$ determined by a matrix $\left(\begin{array}{lll}1 & 1 & 2 \\ 1 & 1 & 3 \\ 2 & 3 & 5\end{array}\right)$ gives an isomorphism from $\Delta_{2}$ to $\Delta_{1}^{*}$, the relation $\Delta_{1}^{*} \simeq \Delta_{2}$ holds.

No. 22. The Newton polytope of $F=F^{\prime}$, which is the convex hull of vertices $(-1,-1,1),(-1,-1,-1),(4,-1,-1)$, and $(-1,1,0)$, coincides with $\Delta_{2}$. Therefore, a pair $\left(\Delta_{1}, \Delta_{F^{\prime}}=\Delta_{2}\right)$ is polytope-dual.

No. 23. The Newton polytope of $F$, which is the convex hull of vertices $(-1,-1,1)$, $(-1,-1,-1),(4,-1,-1)$, and $(-1,1,0)$, coincides with $\Delta_{2}$. The Newton polytope of $F^{\prime}$, which is the convex hull of vertices $(-1,-1,1),(-1,0,-1),(4,-1,-1)$, and $(-1,1,0)$, is not reflexive. Indeed, the polytope $\Delta_{F^{\prime}}$ contains faces $\Gamma_{1}$ spanned by $(-1,-1,1),(-1,0,-1)$, and $(4,-1,-1)$, and $\Gamma_{2}$ spanned by $(-1,1,0)$, $(-1,0,-1)$, and $(4,-1,-1)$, of which the polar duals are non-integral vertices $1 / 7(2,10,5)$, and $1 / 4(-1,-5,5)$, respectively. We have to replace these faces by unions of some sections. For $\Gamma_{1}$, we consider the following try-outs:

First try-out Consider a section $\Gamma_{1}^{\prime}$ spanned by $(-1,-1,1),(-1,0,-1)$, and $(a,-1,-1)$ with $a=3,2,1,0,-1$. The polar dual of $\Gamma_{1}^{\prime}$ is a vertex $v_{1}=$ $1 /(a+3)(2,2(a+1), a+1)$. The vertex $v_{1}$ is a lattice point if and only if $a=-1$.

Second try-out Consider a section $\Gamma_{1}^{\prime \prime}$ spanned by $(-1,-1,0),(-1,0,-1)$, and $(4,-1,-1)$. The polar dual of $\Gamma_{1}^{\prime \prime}$ is a non-integral vertex $1 / 6(1,5,5)$. Thus, it is impossible to use this section.

For $\Gamma_{2}$, consider a section $\Gamma_{2}^{\prime}$ spanned by $(-1,1,0),(4,-1,-1)$, and $(-1, b,-1)$ with $b=3,2,1$. The polar dual of the section $\Gamma_{2}^{\prime}$ is a vertex $v=1 /(b-4)(b+$ $1,5,5(b-1))$. The vertex $v$ is a lattice point if and only if $b=3$.

Therefore, a pair $\left(\Delta_{F}=\Delta_{2}, \Delta_{1}\right)$ is polytope-dual.

No. 24. The Newton polytope of $F=F^{\prime}$, which is the convex hull of vertices $(-1,-1,1),(-1,0,-1),(4,-1,-1)$, and $(-1,1,0)$, is not reflexive. It is observed that one might take a polytope with vertices $(-1,2,-1),(0,2,-1)$ and $(-1,-1,-1)$ instead of faces spanned by vertices $(-1,1,0),(-1,0,-1),(4,-1,-1)$, and $(-1,-1,1),(-1,0,-1),(4,-1,-1)$, namely, $\Delta^{(71)}$. 
In the polytope $\Delta^{(71)}$, the vertex $(-1,-1,-1)$ is adjacent to three other vertices: vertex $(-1,-1,1)$ with an edge $e_{1}$, vertex $(-1,2,-1)$ with an edge $e_{2}$, and vertex $(4,-1,-1)$ with an edge $e_{3}$. On the edges $e_{1}, e_{2}, e_{3}$, respectively, there are one, two, and four inner lattice points. Thus, the polar dual polytope $\Delta^{(71)^{*}}$ must contain a triangle as a two-dimensional face that is adjacent to other two-dimensional faces with inner lattice points one, two, and four. However, it is easily observed that there is no such a configuration in the polytope $\Delta^{(71)^{*}}$. Thus, $\Delta^{(71)}$ is not self-dual, and there does not exist a polytope-dual pair.

\subsection{No. 25}

We claim that there exist two polytope-dual pairs. Take a basis $\left\{e_{1}^{(n)}, e_{2}^{(n)}, e_{3}^{(n)}\right\}$ of lattices $M_{n}$ for $n=37,58$ by

$$
\begin{array}{ll}
e_{1}^{(37)}=(-3,1,0,0), \quad e_{2}^{(37)}=(-4,0,1,0), & e_{3}^{(37)}=(-8,0,0,1), \\
e_{1}^{(58)}=(-4,1,0,0), \quad e_{2}^{(58)}=(-5,0,1,0), & e_{3}^{(58)}=(-6,0,0,1) .
\end{array}
$$

Define polytopes $\Delta_{1}, \Delta_{1}^{\prime}, \Delta_{2}$ and $\Delta_{2}^{\prime}$ by

$$
\begin{aligned}
& \Delta_{1}:=\operatorname{Conv}\left\{\begin{array}{l}
(-1,1,0),(-1,-1,0), \\
(-1,-1,-1),(3,-1,-1),(0,-1,1)
\end{array}\right\}, \\
& \Delta_{1}^{\prime}:=\Delta^{(37)}=\operatorname{Conv}\left\{\begin{array}{l}
(-1,-1,1),(-1,-1,-1), \\
(4,-1,-1),(3,0,-1),(-1,3,-1)
\end{array}\right\}, \\
& \Delta_{2}:=\operatorname{Conv}\left\{\begin{array}{l}
(-1,1,0),(-1,-1,1), \\
(-1,-1,-1),(3,-1,-1),(0,-1,1)
\end{array}\right\}, \\
& \Delta_{2}^{\prime}:=\operatorname{Conv}\left\{\begin{array}{l}
(-1,-1,1),(-1,-1,-1), \\
(3,-1,-1),(3,0,-1),(-1,3,-1)
\end{array}\right\} .
\end{aligned}
$$

Since the polar dual polytopes $\Delta_{1}^{*}$ and $\Delta_{2}^{*}$ of $\Delta_{1}$ and $\Delta_{2}$ are the convex hulls of vertices $(1,0,0),(0,1,0),(0,-1,2),(1,0,-1)$, and $(-4,-5,-6)$, respectively, $(1,0,0),(0,1,0),(0,-1,2),(0,-1,-2)$, and $(-4,-5,-6)$, and the linear map of $\mathbb{R}^{3}$ determined by a matrix $\left(\begin{array}{lll}1 & 1 & 1 \\ 1 & 1 & 2 \\ 2 & 3 & 3\end{array}\right)$ gives isomorphisms from $\Delta_{1}^{\prime}$ to $\Delta_{1}^{*}$, and from $\Delta_{2}^{\prime}$ to $\Delta_{2}^{*}$, the relations $\Delta_{1}^{*} \simeq \Delta_{1}^{\prime}$ and $\Delta_{2}^{*} \simeq \Delta_{2}^{\prime}$ hold.

The Newton polytopes of $F$, which is the convex hull of vertices $(0,-1,1)$, $(-1,-1,-1),(3,-1,-1)$, and $(-1,1,0)$, is not reflexive. Indeed, the polytope $\Delta_{F}$ contains a face $\Gamma$ spanned by $(-1,1,0),(-1,-1,-1)$, and $(0,-1,1)$ of which the polar dual is a non-integral vertex $1 / 3(4,1,-2)$. The face $\Gamma$ has to be replaced by a union of other faces. In order to do this, consider a section $\Gamma^{\prime}$ spanned by $(-1,1,0),(0,-1,1)$, and $(-1,-1, a)$ with $a=0,1$. The polar dual of $\Gamma^{\prime}$ is a vertex $v=1 /(a-2)(2(a-1), a, 2)$. The vertex $v$ is a lattice point if and only if $a=0,1$. Moreover, we note that it is also possible to use a vertex $\left(-1, a^{\prime},-1\right)$ with $a^{\prime}=1,2$ instead of a face spanned by vertices $(0,-1,1),(-1,-1,-1)$, and $(-1,1,0)$ which follows from a direct computation. 
The Newton polytopes of $F^{\prime}$, which is the convex hull of vertices $(-1,-1,1)$, $(-1,-1,-1),(3,0,-1)$, and $(-1,1,0)$, is not reflexive. Indeed, the polytope $\Delta_{F^{\prime}}$ contains faces $\Gamma_{1}$ spanned by $(-1,-1,1),(-1,-1,-1)$, and $(3,0,-1)$, and $\Gamma_{2}$ spanned by $(-1,1,0),(-1,-1,-1)$, and $(3,0,-1)$, of which the polar duals are respectively non-integral vertices $1 / 3(-1,4,0)$ and $1 / 5(1,-4,8)$. The faces $\Gamma_{1}$ and $\Gamma_{2}$ have to be replaced by unions of other sections.

For $\Gamma_{1}$, consider a section $\Gamma_{1}^{\prime}$ spanned by $(-1,-1,-1),(3,0,-1)$, and $(b,-1,-1)$ with $b=4,3,2,1,0$. The polar dual of $\Gamma_{1}^{\prime}$ is a vertex $v_{1}=1 /(b-$ $5)(2,2(b-3), b+1)$. The vertex $v_{1}$ is a lattice point if and only if $b=3,4$.

For $\Gamma_{2}$, consider a section $\Gamma_{2}^{\prime}$ spanned by $(-1,1,0),(3,0,-1)$, and $\left(-1, b^{\prime},-1\right)$ with $b^{\prime}=3,2,1,0$. The polar dual of $\Gamma_{2}^{\prime}$ is a vertex $v_{1}=1 /\left(b^{\prime}-4\right)\left(b^{\prime}, 4,4\left(b^{\prime}-1\right)\right)$. The vertex $v_{2}$ is a lattice point if and only if $b^{\prime}=0,3,2$.

Therefore, there are two polytope-dual pairs, that is, if $\left(a, b, b^{\prime}\right)=(0,4,3)$, then, $\left(\Delta_{1}, \Delta_{1}^{\prime}\right)$, and if $\left(a, b, b^{\prime}\right)=(1,3,3)$, then, $\left(\Delta_{2}, \Delta_{2}^{\prime}\right)$.

\subsection{No. 26}

We claim that there exist four polytope-dual pairs. Take a basis $\left\{e_{1}^{(87)}, e_{2}^{(87)}, e_{3}^{(87)}\right\}$ of a lattice $M_{87}$ by

$$
e_{1}^{(87)}=(-3,1,0,0), \quad e_{2}^{(37)}=(-4,0,1,0), \quad e_{3}^{(37)}=(-5,0,0,1) .
$$

Define polytopes $\Delta_{i}, \Delta_{i}^{\prime}$ for $i=1,2,3,4$ by

$$
\begin{aligned}
& \Delta_{1}:=\operatorname{Conv}\left\{\begin{array}{l}
(-1,-1,1),(-1,1,0),(0,-1,1), \\
(-1,-1,-1),(2,-1,-1),(2,0)-1),(-1,0,-1)
\end{array}\right\}, \\
& \Delta_{1}^{\prime}:=\operatorname{Conv}\left\{\begin{array}{l}
(-1,-1,1),(-1,1,0),(0,-1,1), \\
(-1,-1,-1),(2,-1,-1),(2,0)-1),(-1,2,-1)
\end{array}\right\}, \\
& \Delta_{2}:=\operatorname{Conv}\left\{\begin{array}{l}
(-1,-1,0),(-1,1,0),(0,-1,1), \\
(-1,-1,-1),(3,-1,-1),(2,0,-1),(-1,0,-1)
\end{array}\right\}, \\
& \Delta_{2}^{\prime}:=\operatorname{Conv}\left\{\begin{array}{l}
(-1,-1,0),(-1,1,0),(0,-1,1), \\
(-1,-1,-1),(3,-1,-1),(2,0,-1),(-1,2,-1)
\end{array}\right\}, \\
& \Delta_{3}:=\operatorname{Conv}\left\{\begin{array}{l}
(-1,-1,0),(-1,1,0),(0,-1,1), \\
(-1,-1,-1),(2,-1,-1),(2,0,-1),(-1,2,-1)
\end{array}\right\}, \\
& \Delta_{3}^{\prime}:=\operatorname{Conv}\left\{\begin{array}{l}
(-1,-1,1),(-1,1,0),(0,-1,1), \\
(-1,-1,-1),(3,-1,-1),(2,0,-1),(-1,0,-1)
\end{array}\right\}, \\
& \Delta_{4}:=\operatorname{Conv}\left\{\begin{array}{l}
(-1,-1,0),(-1,1,0),(0,-1,1), \\
(-1,-1,-1),(2,-1,-1),(2,0,-1),(-1,0,-1)
\end{array}\right\}, \\
& \left.\Delta_{4}^{\prime}:=\Delta^{(87)}=\operatorname{Conv} \begin{array}{l}
(-1,-1,1),(-1,1,0),(0,-1,1), \\
(-1,2,-1)
\end{array}\right\} .
\end{aligned}
$$


Since the polar dual polytopes $\Delta_{i}^{*}, i=1,2,3,4$ are respectively given as follows:

$$
\begin{aligned}
& \Delta_{1}^{*}:=\operatorname{Conv}\left\{\begin{array}{l}
(1,0,0),(0,1,0),(0,0,1),(0,-1,-2), \\
(-1,0,-1),(0,-1,1),(-3,-4,-5)
\end{array}\right\}, \\
& \Delta_{2}^{*}:=\operatorname{Conv}\left\{\begin{array}{l}
(1,0,0),(0,1,0),(0,0,1),(1,0,-1), \\
(-2,-2,-3),(0,-1,1),(-3,-4,-5)
\end{array}\right\}, \\
& \Delta_{3}^{*}:=\operatorname{Conv}\left\{\begin{array}{l}
(1,0,0),(0,1,0),(0,0,1),(1,0,-1), \\
(-1,0,-1),(-2,-3,-3),(-3,-4,-5)
\end{array}\right\}, \\
& \Delta_{4}^{*}:=\operatorname{Conv}\left\{\begin{array}{l}
(1,0,0),(0,1,0),(0,0,1),(1,0,-1), \\
(-1,0,-1),(0,-1,1),(-3,-4,-5)
\end{array}\right\},
\end{aligned}
$$

and the linear map of $\mathbb{R}^{3}$ determined by a matrix $\left(\begin{array}{lll}1 & 1 & 1 \\ 1 & 1 & 2 \\ 1 & 2 & 2\end{array}\right)$ gives isomorphisms from $\Delta_{i}^{\prime}$ to $\Delta_{i}^{*}, i=1,2,3,4$, the relations $\Delta_{i}^{*} \simeq \Delta_{i}^{\prime}, i=1,2,3,4$ hold.

The Newton polytope of $F=F^{\prime}$, which is the convex hull of vertices $(0,-1,1)$, $(-1,-1,-1),(2,0,-1)$, and $(-1,1,0)$, is not reflexive. Indeed, the polytope $\Delta_{F}$ contains faces $\Gamma_{1}$ spanned by $(-1,1,0),(-1,-1,-1)$, and $(0,-1,1), \Gamma_{2}$ spanned by $(0,-1,1),(-1,-1,-1)$, and $(2,0,-1)$, and $\Gamma_{3}$ spanned by $(-1,1,0)$, $(-1,-1,-1)$, and $(2,0,-1)$, of which the polar duals are respectively non-integral vertices $1 / 3(4,1,-2), 1 / 5(-2,6,1)$, and $1 / 4(1,-3,6)$. These faces have to be replaced by unions of other sections.

For $\Gamma_{1}$, consider a section $\Gamma_{1}^{\prime}$ spanned by $(-1,1,0),(0,-1,1)$, and $(-1,-1, a)$ with $a=0,1$. The polar dual of $\Gamma_{1}^{\prime}$ is a vertex $v_{1}=1 /(a-2)(2(a-1), a, 2)$. The vertex $v_{1}$ is a lattice point if and only if $a=0,1$.

For $\Gamma_{2}$, consider a section $\Gamma_{2}^{\prime}$ spanned by $(2,0,-1),(0,-1,1)$, and $(b,-1,-1)$ with $b=3,2,1,0$. The polar dual of $\Gamma_{2}^{\prime}$ is a vertex $v_{2}=1 /(b-4)(2,2(b-2), b)$. The vertex $v_{2}$ is a lattice point if and only if $b=2,3$.

For $\Gamma_{3}$, consider a section $\Gamma_{3}^{\prime}$ spanned by $(-1,1,0),(2,0,-1)$, and $(-1, c,-1)$ with $c=2,1,0$. The polar dual of $\Gamma_{3}^{\prime}$ is a vertex $v_{3}=1 /(c-3)(c, 3,3(c-1))$. The vertex $v_{3}$ is a lattice point if and only if $c=0,2$.

Therefore, there are four polytope-dual pairs, that is, if $(a, b, c)=(1,2,0)$ for one side, and $(a, b, c)=(1,2,2)$ for the other, then, $\left(\Delta_{1}, \Delta_{1}^{\prime}\right)$; if $(a, b, c)=(0,3,0)$ for one side, and $(a, b, c)=(0,3,2)$ for the other, then, $\left(\Delta_{2}, \Delta_{2}^{\prime}\right)$; if $(a, b, c)=(0,2,2)$ for one side, and $(a, b, c)=(1,3,0)$ for the other, then, $\left(\Delta_{3}, \Delta_{3}^{\prime}\right)$; and if $(a, b, c)=$ $(0,2,0)$ for one side, and $(a, b, c)=(1,3,2)$ for the other, then, $\left(\Delta_{4}, \Delta_{4}^{\prime}\right)$.

\subsection{No. 27}

We claim that there does not exist a polytope-dual pair. Take a basis $\left\{e_{1}^{(4)}, e_{2}^{(4)}, e_{3}^{(4)}\right\}$ of a lattice $M_{4}$ by

$$
e_{1}^{(4)}=(-3,1,0,0), \quad e_{2}^{(4)}=(-4,0,1,0), \quad e_{3}^{(4)}=(-4,0,0,1) .
$$

By a direct computation, one has $\Delta^{(4)^{*}} \nsucceq \Delta^{(4)}$, which we say not self-dual. 
It is observed that there exist four invertible projectivisations

$$
\begin{aligned}
& F_{1}=F_{1}^{\prime}=X^{4}+Y^{3}+Z^{3}+W^{12}, \quad F_{2}=F_{2}^{\prime}=X^{4}+Y^{3}+Z^{3}+W^{8} Z \\
& F_{3}=F_{3}^{\prime}=X^{4}+Y^{3}+Z^{3}+W^{8} Y, F_{4}=F_{4}^{\prime}=X^{4}+Y^{3}+Z^{3}+W^{9} X
\end{aligned}
$$

The Newton polytope of $F_{1}=F_{1}^{\prime}$, which is the convex hull of vertices $(-1,-1,2)$, $(-1,-1,-1),(3,-1,-1)$, and $(-1,2,-1)$, coincide with $\Delta^{(4)}$.

Since $F_{2}$ and $F_{3}$ are symmetric in variables $Y$ and $Z$, we only treat with $F_{2}$. The Newton polytope of $F_{2}=F_{2}^{\prime}$, which is the convex hull of vertices $(-1,-1,2)$, $(-1,-1,0),(3,-1,-1)$, and $(-1,2,-1)$, is not reflexive. Indeed, the polytope $\Delta_{F_{2}}$ contains a face $\Gamma$ spanned by $(-1,-1,0),(-1,2,-1)$, and $(3,-1,-1)$ of which the polar dual is non-integral vertex $1 / 7(3,4,12)$. The face $\Gamma$ has to be replaced by a union of some sections.

First try-out Consider a face $\Gamma^{\prime}$ spanned by $(-1,-1,0),(3,-1,-1)$, and $(-1, a,-1)$ with $a=1,0,-1$. The polar dual of $\Gamma^{\prime}$ is a vertex $v^{\prime}=1 /(a+5)(a+$ $1,4,4(a+1))$. The vertex $v^{\prime}$ is a lattice point if and only if $a=-1$.

Second try-out Consider a face $\Gamma^{\prime \prime}$ spanned by $(-1,-1,0),(-1,2,-1)$, and $(b,-1,-1)$ with $b=2,1,0,-1$. The polar dual of $\Gamma^{\prime \prime}$ is a vertex $v^{\prime \prime}=1 /(b+$ 4) $(3, b+1,3(b+1))$. The vertex $v^{\prime \prime}$ is a lattice point if and only if $b=-1$.

Third try-out Consider a face $\Gamma^{\prime \prime \prime}$ spanned by $(-1,-1,0),(-1, a,-1)$, and $(b,-1,-1)$ with $a=1,0,-1$ and $b=2,1,0,-1$. Note that $a=-1$ and $b=-1$ simultaneously occur. If $a=b=-1$, then, the resulting polytope is none other than $\Delta^{(4)}$. Otherwise, the polar dual of $\Gamma^{\prime \prime \prime}$ is a vertex $v^{\prime \prime \prime}=1 /(a+b+2)(a+1, b+$ $1,(a+1)(b+1))$, which is never a lattice point.

Therefore, the only reflexive polytope $\Delta$ such that $\Delta_{F_{2}} \subset \Delta$ is $\Delta^{(4)}$.

The Newton polytope of $F_{4}=F_{4}^{\prime}$, which is the convex hull of vertices $(-1,-1,2)$, $(0,-1,-1),(3,-1,-1)$, and $(-1,2,-1)$, is not reflexive. Indeed, the polytope $\Delta_{F_{4}}$ contains a face $\Gamma$ spanned by $(-1,-1,2),(0,-1,-1)$, and $(-1,2,-1)$, of which the polar dual is a non-integral vertex $1 / 2(3,1,1)$. The face $\Gamma$ has to be replaced by a union of other sections. Consider a section $\Gamma^{\prime}$ spanned by $(-1,-1,2),(0,-1,-1)$, and $(-1, a,-1)$ with $a=1,0,-1$. The polar dual of $\Gamma^{\prime}$ is a vertex $v^{\prime}=1 /(a+$ 4) $(3(a+1), 3, a+1)$. The vertex $v^{\prime}$ is a lattice point if and only if $a=-1$. Therefore, the only reflexive polytope $\Delta$ with $\Delta_{F_{4}} \subset \Delta$ is $\Delta^{(4)}$.

\subsection{No. 28-No. 29}

We claim that there exist two polytope-dual pairs for Nos. 28 and 29. Take a basis $\left\{e_{1}^{(n)}, e_{2}^{(n)}, e_{3}^{(n)}\right\}$ of lattices $M_{n}$ for $n=12,27$ and 49 by

$$
\begin{aligned}
& e_{1}^{(12)}=(-2,1,0,0), \quad e_{2}^{(12)}=(-6,0,1,0), \quad e_{3}^{(12)}=(-9,0,0,1), \\
& e_{1}^{(27)}=-(1,-7,1,1), e_{2}^{(27)}=-(1,1,-2,1), e_{3}^{(27)}=-(0,1,1,-1), \\
& e_{1}^{(49)}=-(0,-7,1,1), e_{2}^{(49)}=-(1,1,-2,1), e_{3}^{(49)}=-(1,1,1,-1) .
\end{aligned}
$$


In Kobayashi and Mase (2012), it is proved that the polytopes $\Delta^{(27)}$ and $\Delta^{(49)}$ are isomorphic to the polytope $\Delta_{(27,49)}$ with vertices $(0,0,1),(1,0,0),(0,1,0)$, and $(-3,-8,-12)$.

Define polytopes $\Delta_{1}, \Delta_{1}^{\prime}, \Delta_{2}$ and $\Delta_{2}^{\prime}$ by

$$
\begin{aligned}
& \Delta_{1}:=\operatorname{Conv}\{(0,0,1),(1,0,0),(0,1,0),(-2,-6,-9)\}, \\
& \Delta_{1}^{\prime}:=\Delta^{(12)}=\operatorname{Conv}\left\{\begin{array}{l}
(-1,-1,1),(-1,-1,-1), \\
(8,-1,-1),(-1,2,-1)
\end{array}\right\}, \\
& \Delta_{2}:=\Delta^{(49)}=\operatorname{Conv}\{(0,0,1),(1,0,0),(0,1,0),(-3,-8,-12)\}, \\
& \Delta_{2}^{\prime}:=\operatorname{Conv}\{(-1,-1,1),(-1,-1,-1),(7,-1,-1),(-1,2,-1)\} .
\end{aligned}
$$

It is direct to see that the relations $\Delta_{1}^{*} \simeq \Delta_{1}^{\prime}$ and $\Delta_{2}^{*} \simeq \Delta_{2}^{\prime}$ hold.

No. 28. The Newton polytope of $F$, which is the convex hull of vertices $(1,0,0)$, $(0,1,0),(0,0,1)$, and $(-2,-6,-9)$, coincides with $\Delta_{1}^{\prime}$. The Newton polytope of $F^{\prime}$, which is the convex hull of vertices $(-1,-1,1),(-1,-1,0),(7,-1,-1)$, and $(-1,2,-1)$, is not reflexive. Indeed, the polytope $\Delta_{F^{\prime}}$ contains a face $\Gamma$ spanned by $(-1,-1,0),(7,-1,-1)$, and $(-1,2,-1)$ of which the polar dual is a non-integral vertex $1 / 11(3,8,24)$. The face $\Gamma$ has to be replaced by a union of other sections.

First try-out Consider a section $\Gamma^{\prime}$ spanned by $(-1,-1,0),(7,-1,-1)$, and $(-1, a,-1)$ with $a=1,0,-1$. The polar dual of $\Gamma^{\prime}$ is a vertex $v^{\prime}=1 /(a+9)(a+$ $1,8,8(a+1))$. The vertex $v^{\prime}$ is a lattice point if and only if $a=-1$.

Second try-out Consider a section $\Gamma^{\prime \prime}$ spanned by $(-1,-1,0),(-1,2,-1)$, and $(b,-1,-1)$ with $b=6,5,4,3,2,1,0,-1$. The polar dual of $\Gamma^{\prime \prime}$ is a vertex $v^{\prime \prime}=$ $1 /(b+4)(3, b+1,3(b+1))$. The vertex $v^{\prime \prime}$ is a lattice point if and only if $b=-1$.

Third try-out Consider a section $\Gamma^{\prime \prime \prime}$ spanned by $(-1,-1,0),(-1, a,-1)$, and $(b,-1,-1)$ with $a=1,0,-1$ and $b=6,5,4,3,2,1,0,-1$. Note that $a=-1$ and $b=-1$ simultaneously occur. If $a=b=-1$, then, the resulting sections have lattice dual vertex. Otherwise, the polar dual of $\Gamma^{\prime \prime \prime}$ is a vertex $v^{\prime \prime \prime}=$ $1 /(a+b+2)(a+1, b+1,(a+1)(b+1))$, which is never a lattice point.

Therefore, there are two polytope-dual pairs : $\left(\Delta_{F}=\Delta_{1}, \Delta_{1}^{\prime}\right)$, and $\left(\Delta_{2}, \Delta_{2}^{\prime}\right)$.

No. 29. The Newton polytope of $F$, which is the convex hull of vertices $(1,0,0)$, $(0,1,0),(0,0,1)$, and $(-2,-6,-9)$, coincides with $\Delta_{1}^{\prime}$. The Newton polytope of $F^{\prime}$, which is the convex hull of vertices $(-1,-1,1),(0,-1,-1),(7,-1,-1)$, and $(-1,2,-1)$, is not reflexive.

Indeed, the polytope $\Delta_{F^{\prime}}$ contains a face $\Gamma$ spanned by $(-1,-1,1),(0,-1,-1)$, and $(-1,2,-1)$, of which the polar dual is a non-integral vertex $1 / 5(6,2,3)$. The face $\Gamma$ has to be replaced by a union of other sections. Consider a section $\Gamma^{\prime}$ spanned by $(-1,-1,1),(0,-1,-1)$, and $(-1, a,-1)$ with $a=1,0,-1$. The polar dual of $\Gamma^{\prime}$ is a vertex $v^{\prime}=1 /(a+3)(2(a+1), 2, a+1)$. The vertex $v^{\prime}$ is a lattice point if and only if $a=-1$.

Note that the polytope $\Delta_{F^{\prime}}$ contains a face spanned by $(-1,-1,1),(7,-1,-1)$, and $(-1,2,-1)$, with which is possible to be replaced by

$$
\operatorname{Conv}\left\{\begin{array}{l}
(-1,-1,1),(7,-1,-1), \\
(8,-1,-1)
\end{array}\right\}+\operatorname{Conv}\left\{\begin{array}{l}
(-1,-1,1),(-1,2,-1), \\
(8,-1,-1)
\end{array}\right\}
$$


or

$$
\operatorname{Conv}\left\{\begin{array}{l}
(-1,-1,1),(7,-1,-1), \\
(5,0,-1)
\end{array}\right\}+\operatorname{Conv}\left\{\begin{array}{l}
(-1,-1,1),(-1,2,-1), \\
(5,0,-1)
\end{array}\right\}
$$

or

$$
\operatorname{Conv}\left\{\begin{array}{l}
(-1,-1,1),(7,-1,-1), \\
(2.1,-1)
\end{array}\right\}+\operatorname{Conv}\left\{\begin{array}{l}
(-1,-1,1),(-1,2,-1), \\
(2,1,-1)
\end{array}\right\} .
$$

In these cases, one has to take $\Delta$ to be a cone of a hexagon with $\Delta^{*}$ to have a configuration of edges that form a lattice of type $T_{9,3,4}$. However, the maximal number of lattice points in an edge of $\Delta^{(12)}$ is 8 , and if we use this edge, we only construct a configuration of edges that form a lattice of type $T_{9,4,4}$. Thus, in such a case, it is impossible to find out a corresponding polytope-dual partner $\Delta^{\prime}$.

Therefore, there are two polytope-dual pairs, that is, $\left(\Delta_{F}=\Delta_{1}, \Delta_{1}^{\prime}\right)$, and $\left(\Delta_{2}, \Delta_{2}^{\prime}\right)$.

\subsection{No. 30}

We claim that there exist two polytope-dual pairs. Take a basis $\left\{e_{1}^{(n)}, e_{2}^{(n)}, e_{3}^{(n)}\right\}$ of lattices $M_{n}$ for $n=40,81$ by

$$
\begin{gathered}
e_{1}^{(40)}=(-2,1,0,0), \quad e_{2}^{(40)}=(-4,0,1,0), \quad e_{3}^{(40)}=(-7,0,0,1), \\
e_{1}^{(81)}=(0,7,-1,-1), \quad e_{2}^{(81)}=(0,-1,2,-1), \quad e_{3}^{(81)}=(-1,-1,-1,1) .
\end{gathered}
$$

Define polytopes $\Delta_{1}, \Delta_{1}^{\prime}, \Delta_{2}, \Delta_{2}^{\prime}$ by

$$
\begin{aligned}
& \Delta_{1}:=\operatorname{Conv}\left\{\begin{array}{l}
(0,1,0),(0,0,1), \\
(1,1,1),(0,-2,-3),(-2,-6,-9)
\end{array}\right\}, \\
& \Delta_{1}^{\prime}:=\Delta^{(40)}=\operatorname{Conv}\left\{\begin{array}{l}
(-1,-1,1),(-1,-1,-1), \\
(6,-1,-1),(0,2,-1),(-1,2,-1)
\end{array}\right\}, \\
& \Delta_{2}:=\operatorname{Conv}\left\{\begin{array}{l}
(0,1,0),(0,0,1), \\
(1,1,1),(1,0,0),(-2,-6,-9)
\end{array}\right\}, \\
& \Delta_{2}^{\prime}:=\operatorname{Conv}\left\{\begin{array}{l}
(-1,-1,1),(-1,-1,-1), \\
(6,-1,-1),(0,2,-1),(-1,1,-1)
\end{array}\right\} .
\end{aligned}
$$

Since the polar dual polytopes $\Delta_{1}^{*}$ and $\Delta_{2}^{*}$ of $\Delta_{1}$ and $\Delta_{2}$ are the convex hulls of vertices $(-1,-1,1),(1,-1,-1),(-2,2,-1),(-1,2,-1),(8,-1,-1)$, resp., $(-1,-1,1),(1,-1,-1),(-1,1,-1),(-1,2,-1),(8,-1,-1)$, and the linear map of $\mathbb{R}^{3}$ determined by a matrix $\left(\begin{array}{ccc}1 & 0 & 0 \\ -1 & 1 & 0 \\ -1 & 0 & 1\end{array}\right)$ gives isomorphisms from $\Delta_{i}^{\prime}$ to $\Delta_{i}^{*}$ for $i=1,2$, the relations $\Delta_{1}^{*} \simeq \Delta_{1}^{\prime}$ and $\Delta_{2}^{*} \simeq \Delta_{2}^{\prime}$ hold.

The Newton polytope of $F$, which is the convex hull of vertices $(1,1,1),(0,1,0)$, $(0,0,1)$, and $(-2,-6,-9)$, is not reflexive. Indeed, the polytope $\Delta_{F}$ contains a face 
spanned by $(0,0,1),(1,1,1)$, and $(-1,-6,-9)$, of which the polar dual is a nonintegral vertex $1 / 2(-5,5,-2)$. The face should be replaced by a sum of some sections.

Consider a section spanned by $(0,0,1),(1,1,1)$, and $(-2+a,-6+2 a,-9+3 a)$ with $a=1,2,3$. The polar dual of this section is a vertex $v=1 /(a-4)(-3 a+$ $10,3 a-10, a-4)$. Thus, the vertex $v$ is a lattice point if and only if $a=2,3$. Namely, one can take a reflexive polytope $\Delta$ to be $\Delta_{1}$, i.e., $a=2$ and $\Delta_{2}$, i.e., $a=3$.

Besides, the Newton polytope of $F^{\prime}$, which is the convex hull of vertices $(-1,-1,1),(-1,0,-1),(5,-1,-1)$, and $(0,2,-1)$, is not reflexive. According to the observation for $\Delta$, we must take two polytopes which are isomorphic to $\Delta_{1}^{*}$ and $\Delta_{2}^{*}$. Indeed, we may take $\Delta_{1}^{\prime}$ and $\Delta_{2}^{\prime}$ that clearly contain $\Delta_{F^{\prime}}$ as a subpolytope.

Therefore, there are two polytope-dual pairs : $\left(\Delta_{1}, \Delta_{1}^{\prime}\right)$, and $\left(\Delta_{2}, \Delta_{2}^{\prime}\right)$.

Note that taking cones over a hexagon as a polytope $\Delta$ is impossible since in each candidate cases, the configurations of lattice points in their polar dual is impossible as a subpolytope of $\Delta^{(40)}$.

\subsection{No. 31}

We claim that there exist five polytope-dual pairs. Take a basis $\left\{e_{1}^{(n)}, e_{2}^{(n)}, e_{3}^{(n)}\right\}$ of lattices $M_{n}$ for $n=11,24$ by

$$
\begin{aligned}
& e_{1}^{(11)}=(2,7,-1,-1), e_{2}^{(11)}=-(1,1,-2,1), e_{3}^{(11)}=-(1,1,1,-1), \\
& e_{1}^{(24)}=(-2,1,0,0), \quad e_{2}^{(24)}=(-4,0,1,0), \quad e_{3}^{(24)}=(-5,0,0,1) .
\end{aligned}
$$

Define polytopes $\Delta_{i}, \Delta_{i}^{\prime}, i=1,2,3,4$ by

$$
\begin{aligned}
& \Delta_{1}:=\Delta^{(11)}=\operatorname{Conv}\{(0,1,0),(0,0,1),(2,2,3),(-3,-8,-12)\}, \\
& \Delta_{1}^{\prime}:=\operatorname{Conv}\{(0,-1,1),(-1,-1,-1),(4,-1,-1),(-1,2,-1)\}, \\
& \Delta_{2}:=\operatorname{Conv}\{(0,1,0),(0,0,1),(2,2,3),(-2,-6,-9)\}, \\
& \Delta_{2}^{\prime}:=\operatorname{Conv}\{(0,-1,1),(-1,-1,-1),(5,-1,-1),(-1,2,-1)\}, \\
& \Delta_{3}:=\operatorname{Conv}\{(0,1,0),(0,0,1),(1,1,2),(0,-2,-3),(-2,-6,-9)\}, \\
& \Delta_{3}^{\prime}:=\Delta^{(24)}=\operatorname{Conv}\left\{\begin{array}{l}
(0,-1,1),(-1,-1,-1), \\
(5,-1,-1),(-1,-1,1),(-1,2,-1)
\end{array}\right\}, \\
& \Delta_{4}:=\operatorname{Conv}\left\{\begin{array}{l}
(0,1,0),(0,0,1),(1,1,2), \\
(0,-2,-3),(-2,-6,-9),(-1,-2,-4)
\end{array}\right\}, \\
& \Delta_{4}^{\prime}:=\operatorname{Conv}\left\{\begin{array}{l}
(0,-1,1),(-1,-1,-1),(4,-1,-1), \\
(3,0,-1),(-1,2,-1),(-1,-1,1)
\end{array}\right\}, \\
& \Delta_{5}:=\operatorname{Conv}\left\{\begin{array}{l}
(0,1,0),(0,0,1),(1,1,2), \\
(0,-2,-3),(-2,-6,-9),(-2,-5,-8)
\end{array}\right\}, \\
& \Delta_{5}^{\prime}:=\operatorname{Conv}\left\{\begin{array}{l}
(0,-1,1),(-1,-1,-1),(4,-1,-1), \\
(1,1,-1),(-1,2,-1),(-1,-1,1)
\end{array}\right\} .
\end{aligned}
$$


Since the polar dual polytopes $\Delta_{i}^{*}$ of $\Delta_{i}, i=1,2,3,4,5$ are respectively the convex hulls of vertices $(-1,-1,1),(2,-1,-1),(-1,2,-1)$, and $(7,-1,-1)$, resp., $(-1,-1,1),(2,-1,-1),(-1,2,-1)$, and $(8,-1,-1)$, resp., $(-1,-1,1)$, $(2,-1,-1), \quad(-1,2,-1), \quad(-2,-1,1), \quad$ and $(8,-1,-1)$, resp., $(-1,-1,1)$, $(2,-1,-1),(-1,2,-1),(-2,-1,1),(5,0,-1)$, and $(7,-1,-1)$, resp. $(-1,-1,1)$, $(2,-1,-1),(-1,2,-1),(-2,-1,1),(2,1,-1)$, and $(7,-1,-1)$, and the linear map of $\mathbb{R}^{3}$ determined by a matrix $\left(\begin{array}{lll}1 & 1 & 1 \\ 1 & 2 & 2 \\ 1 & 2 & 3\end{array}\right)$ gives isomorphisms from $\Delta_{i}^{\prime}$ to $\Delta_{i}^{*}$ for $i=1,2,3,4,5$, the relations $\Delta_{i}^{*} \simeq \Delta_{i}^{\prime}, i=1,2,3,4,5$ hold.

The Newton polytope of $F$, which is the convex hull of vertices $(0,1,0),(0,0,1)$, $(1,1,2),(-2,-6,-9)$, is not reflexive. Indeed, the polytope $\Delta_{F}$ contains a face spanned by $(1,1,2),(0,1,0)$, and $(-2,-6,-9)$, of which the polar dual is a nonintegral vertex $1 / 5(-14,-5,7)$. Consider a section spanned by $(1,1,2),(0,1,0)$, and $(-2+a,-6+2 a,-9+3 a)$ with $a=1,2,3,4$. The polar dual of this section is a vertex $v=1 /(a-5)(2(2 a-7), a-5,2 a-7)$. The vertex $v$ is a lattice point if and only if $a=2,4$. Note that we can use a vertex $(-1,-2,-4)$ or $(-2,-5,-8)$ and replace the face spanned by $(0,01),(0,1,0)$ and $(-2,-6,-9)$ by either the sum

$$
\operatorname{Conv}\left\{\begin{array}{c}
(0,0,1),(-2,-6,-9), \\
(-1,-2,-4)
\end{array}\right\}+\operatorname{Conv}\left\{\begin{array}{c}
(0,0,1),(0,1,0), \\
(-1,-2,-4)
\end{array}\right\}
$$

or

$$
\operatorname{Conv}\left\{\begin{array}{c}
(0,0,1),(-2,-6,-9), \\
(-2,-5,-8)
\end{array}\right\}+\operatorname{Conv}\left\{\begin{array}{c}
(0,0,1),(0,1,0) \\
(-2,-5,-8)
\end{array}\right\}
$$

However, it is impossible to consider as $\Delta$ to be

$$
\operatorname{Conv}\{(0,01),(-2,-6,-9),(2,2,3),(0,1,0), v\},
$$

where $v=(-1,-2,-4)$ or $(-2,-5,-8)$ becase the polar dual polytope should contain three incident edges of type $T_{2,3,6}$ without an edge with two lattice points, which is impossible.

Therefore, by the observation, there are five polytope-dual pairs, that is, $\left(\Delta_{1}, \Delta_{F^{\prime}}=\right.$ $\left.\Delta_{1}^{\prime}\right),\left(\Delta_{2}, \Delta_{2}^{\prime}\right),\left(\Delta_{3}, \Delta_{3}^{\prime}\right),\left(\Delta_{4}, \Delta_{4}^{\prime}\right)$, and $\left(\Delta_{5}, \Delta_{5}^{\prime}\right)$.

\subsection{No. 32-No. 34}

We claim that there exist two polytope-dual pairs for Nos. 32 to 34 . Take a basis $\left\{e_{1}^{(n)}, e_{2}^{(n)}, e_{3}^{(n)}\right\}$ of lattices $M_{n}$ with for $n=6,26,34$, and 76 by

$$
\begin{aligned}
& e_{1}^{(6)}=(-2,1,0,0), \quad e_{2}^{(6)}=(-2,0,1,0), \quad e_{3}^{(6)}=(-5,0,0,1), \\
& e_{1}^{(26)}=-(1,-4,1,1), e_{2}^{(26)}=-(1,1,-3,1), e_{3}^{(26)}=-(0,1,1,-1), \\
& e_{1}^{(34)}=-(1,-4,1,1), e_{2}^{(34)}=-(0,1,-3,1), e_{3}^{(34)}=-(1,1,1,-1), \\
& e_{1}^{(76)}=(1,1,1,-1), \quad e_{2}^{(76)}=(4,1,0,-1), \quad e_{3}^{(76)}=(9,-1,0,-1) .
\end{aligned}
$$


In Kobayashi and Mase (2012), it is proved that the polytopes $\Delta^{(26)}$ and $\Delta^{(34)}$ are isomorphic to the polytope with vertices $(1,0,0),(0,1,0),(0,0,1)$, and $(-4,-5,-10)$.

Define polytopes $\Delta_{1}, \tilde{\Delta}_{1}, \Delta_{1}^{\prime}$, and $\Delta_{2}, \tilde{\Delta}_{2}, \Delta_{2}^{\prime}$ by

$$
\begin{aligned}
& \Delta_{1}:=\operatorname{Conv}\{(1,0,0),(0,1,0),(0,0,1),(-2,-2,-5)\}, \\
& \tilde{\Delta}_{1}:=\operatorname{Conv}\{(0,0,-1),(2,0,-1),(2,-1,0),(-3,1,3)\}, \\
& \Delta_{1}^{\prime}:=\Delta^{(6)}=\operatorname{Conv}\left\{\begin{array}{l}
(-1,-1,1),(-1,-1,-1), \\
(4,-1,-1),(-1,4,-1)
\end{array}\right\}, \\
& \Delta_{2}:=\operatorname{Conv}\left\{\begin{array}{l}
(1,0,0),(0,1,0),(0,0,1), \\
(-2,-2,-5),(-a,-(a+1),-2(a+1))
\end{array}\right\}, \\
& \tilde{\Delta}_{2}:=\operatorname{Conv}\left\{\begin{array}{l}
(0,0,-1),(2,0,-1),(2,-1,0), \\
(-3,1,3),(a-2,1,-a+2)
\end{array}\right\}, \\
& \Delta_{2}^{\prime}:=\operatorname{Conv}\left\{\begin{array}{l}
(-1,-1,1),(-1,-1,-1),(4,-1,-1), \\
(-1,3,-1),(a, 3-a,-1)
\end{array}\right\}, \\
& \text { here, } \quad \begin{array}{l}
a=3,2,1,0 .
\end{array}
\end{aligned}
$$

Since the polar dual polytopes $\Delta_{1}^{*}$ and $\tilde{\Delta}_{1}^{*}$ of $\Delta_{1}$ and $\tilde{\Delta}_{1}$ are the convex hulls of vertices $(-1,-1,1),(-1,-1,-1),(4,-1,-1)$, and $(-1,4,-1)$, respectively, $(-1,-1,-1),(0,1,1),(0,-4,1)$, and $(5,11,1)$, and the linear map of $\mathbb{R}^{3}$ determined by a matrix $\left(\begin{array}{ccc}-1 & -3 & 0 \\ -1 & -2 & 0 \\ -3 & -6 & -1\end{array}\right)$ gives an isomorphism from $\Delta_{1}^{\prime}$ to $\tilde{\Delta}_{1}^{*}$, the relations $\Delta_{1}^{*}=\Delta_{1}^{\prime} \simeq \tilde{\Delta}_{1}^{*}$ hold. Moreover, Since the polar dual polytopes $\Delta_{2}^{*}$ and $\tilde{\Delta}_{2}^{*}$ of $\Delta_{2}$ and $\tilde{\Delta}_{2}$ are convex hulls of the vertices $(-1,-1,1),(-1,-1,-1),(4,-1,-1)$, $(a, 3-a,-1)$, and $(-1,3,-1)$, respectively, $(-1,-1,-1),(0,1,1),(5,11,1)$, $(1,-1,1)$, and $(0, a-3,1)$, and the linear map of $\mathbb{R}^{3}$ determined by a matrix $\left(\begin{array}{ccc}-1 & -2 & 0 \\ -1 & -3 & 0 \\ -3 & -6 & -1\end{array}\right)$ gives an isomorphism from $\Delta_{2}^{\prime}$ to $\tilde{\Delta}_{2}^{*}$, the relations $\Delta_{2}^{*}=\Delta_{2}^{\prime} \simeq \tilde{\Delta}_{2}^{*}$ hold.

No. 32. The Newton polytope of $F$, which is the convex hull of vertices $(1,0,0)$, $(0,1,0),(0,0,1)$, and $(-2,-2,-5)$, coincides with $\Delta_{1}$. Besides, the Newton polytope of $F^{\prime}$, which is the convex hull of vertices $(-1,-1,0),(-1,3,-1),(-1,-1,1)$, and $(4,-1,-1)$, is not reflexive. Indeed, the polytope $\Delta_{F^{\prime}}$ contains a face spanned by $(-1,-1,1),(-1,-1,0)$, and $(-1,3,-1)$, of which the polar dual is a nonintegral vertex $1 / 9(4,5,20)$. Thus, we consider a section spanned by $(-1,-1,0)$, $(4,-1,-1)$, and $(-1, a,-1)$ with $a=1-1,0,1,2$. The polar dual of this section is a vertex $v=1 /(a+6)(a+1,5,5(a+1))$. Thus, the vertex $v$ is a lattice point if and only if $a=-1$. Therefore, there are two polytope-dual pairs, that is, $\left(\Delta_{F}=\Delta_{1}, \Delta_{1}^{\prime}\right)$, and $\left(\Delta_{2}, \Delta_{2}^{\prime}\right)$.

No. 33. The Newton polytope of $F$, which is the convex hull of vertices $(1,0,0)$, $(0,1,0),(0,0,1)$, and $(-2,-2,-5)$, coincides with $\Delta_{1}$. Besides, the Newton polytope of $F^{\prime}$, which is the convex hull of vertices $(-1,0,-1),(-1,3,-1),(-1,-1,1)$, and $(4,-1,-1)$, is not reflexive. Indeed, the polytope $\Delta_{F^{\prime}}$ contains a face spanned by $(-1,-1,1),(-1,0,-1)$, and $(4,-1,-1)$, of which the polar dual is a nonintegral vertex $1 / 7(2,10,5)$. Thus, we consider a section spanned by $(-1,-1,1)$, 
$(-1,0,-1)$, and $(a,-1,-1)$ with $a=1-1,0,1,2,3$. The polar dual of this section is a vertex $v=1 /(a+3)(2,2(a+1), a+1)$. Thus, the vertex $v$ is a lattice point if and only if $a=-1$. Therefore, there are two polytope-dual pairs, that is, $\left(\Delta_{F}=\Delta_{1}, \Delta_{1}^{\prime}\right)$, and $\left(\Delta_{2}, \Delta_{2}^{\prime}\right)$.

No. 34. The Newton polytope of $F$, which is the convex hull of vertices $(0,0,-1)$, $(2,0,-1),(2,-1,0)$, and $(-3,1,3)$, coincides with $\tilde{\Delta}_{1}$. Besides, the Newton polytope of $F^{\prime}$, which is the convex hull of vertices $(-1,0,-1),(3,-1,-1),(0,3,-1)$, and $(-1,-1,1)$, is not reflexive. Indeed, the polytope $\Delta_{F^{\prime}}$ contains two faces $\Gamma_{1}$ spanned by $(-1,-1,1),(-1,0,-1)$, and $(3,-1,-1)$, of which the polar dual is $1 / 3(1,4,2)$, and $\Gamma_{2}$ spanned by $(-1,-1,1),(-1,0,-1)$, and $(0,3,-1)$, of which the polar dual is $1 / 5(6,-2,-1)$. The face $\Gamma_{1}$ is replaced by a sum of other sections as is discussed in No.33. For the face $\Gamma_{2}$, consider a section spanned by $(-1,-1,1)$, $(0,3,-1)$, and $(-1, a,-1)$ with $a=1,2,3,4$. The polar dual of this section is a vertex $v=1 /(a-5)(2(a-3), 2, a+1)$. The vertex $v$ is a lattice point if and only if $a=3,4$. Therefore, there are two polytope-dual pairs, that is, $\left(\Delta_{F}=\tilde{\Delta}_{1}, \Delta_{1}^{\prime}\right)$, and $\left(\tilde{\Delta}_{2}, \Delta_{2}^{\prime}\right)$.

\subsection{No. 35-No. 37}

We claim that there exists a unique polytope-dual pair for Nos. 35 to 37 . Take a basis $\left\{e_{1}^{(n)}, e_{2}^{(n)}, e_{3}^{(n)}\right\}$ lattices $M_{n}$ for $n=10,46,65$, and 80 by

$$
\begin{aligned}
& e_{1}^{(10)}=(-1,1,0,0), \quad e_{2}^{(10)}=(-4,0,1,0), \quad e_{3}^{(10)}=(-6,0,0,1), \\
& e_{1}^{(46)}=(1,1,1,-1), \quad e_{2}^{(46)}=(2,2,-1,0), \quad e_{3}^{(46)}=(11,0,-1,-1), \\
& e_{1}^{(65)}=(1,0,1,-1), \quad e_{2}^{(65)}=(2,1,-1,0), \quad e_{3}^{(65)}=(10,-1,-1,-1), \\
& e_{1}^{(80)}=(1,1,1,-1), \quad e_{2}^{(80)}=(2,1,-1,0), \quad e_{3}^{(80)}=(10,-1,-1,-1) .
\end{aligned}
$$

In Kobayashi and Mase (2012), it is proved that the polytopes $\Delta^{(46)}, \Delta^{(65)}$ and $\Delta^{(80)}$ are isomorphic to the polytope $\Delta_{(46,65,80)}$ with vertices $(-1,0,0),(1,-1,0)$, $(0,0,1)$, and $(2,4,-1)$. Let a polytope $\Delta_{1}$ be $\Delta_{(46,65,80)}$ and $\Delta_{1}^{\prime}$ be the polytope $\Delta^{(10)}$ which is the convex hull of vertices $(-1,-1,1),(-1,-1,-1),(11,-1,-1)$, and $(-1,2,-1)$. Since the polar dual polytope $\Delta_{1}^{*}$ of $\Delta_{1}$ is the convex hull of vertices $(1,-1,-1),(1,2,11),(1,2,-1)$, and $(-1,0,-1)$, and the linear map of $\mathbb{R}^{3}$ determined by a matrix $\left(\begin{array}{ccc}0 & 0 & -1 \\ 0 & -1 & -4 \\ -1 & -1 & -6\end{array}\right)$ gives an isomorphism from $\Delta_{1}^{\prime}$ to $\Delta_{1}^{*}$, the relation $\Delta_{1}^{*} \simeq \Delta_{1}^{\prime}$ holds.

For each case of Nos. 35, 36, 37, the Newton polytope of $F$, which is the convex hull of vertices $(-1,0,0),(1,-1,0),(0,0,1)$, and $(2,4,-1)$, coincides with $\Delta_{1}=$ $\Delta_{(46,65,80)}$. Thus, there exists only one reflexive polytope $\Delta$ such that $\Delta_{F} \subset \Delta \subset$ $\Delta_{(46,65,80)}$, which is none other than $\Delta_{1}$. The polytope $\Delta_{1}^{\prime}$ no doubt satisfies $\Delta_{F^{\prime}} \subset$ $\Delta_{1}^{\prime}=\Delta^{(10)}$. Therefore, there exists only one polytope-dual pair $\left(\Delta_{1}, \Delta_{1}^{\prime}\right)$. 


\subsection{No. 38-No. 40}

We claim that there exist two polytope-dual pairs for Nos. 38 and 40, and that none for No. 39. Take a basis $\left\{e_{1}^{(n)}, e_{2}^{(n)}, e_{3}^{(n)}\right\}$ of lattices $M_{n}$ for $n=42,68,83$, and 92 by

$$
\begin{aligned}
& e_{1}^{(42)}=(-1,1,0,0), \quad e_{2}^{(42)}=(-3,0,1,0), \quad e_{3}^{(42)}=(-5,0,0,1), \\
& e_{1}^{(68)}=(1,0,1,-1), \quad e_{2}^{(68)}=(3,1,0,-1), \quad e_{3}^{(68)}=(9,-1,-1,-1), \\
& e_{1}^{(83)}=(1,1,1,-1), \quad e_{2}^{(83)}=(3,3,0,-1), \quad e_{3}^{(83)}=(10,1,-1,-1), \\
& e_{1}^{(92)}=(1,1,1,-1), \quad e_{2}^{(92)}=(3,2,0,-1), \quad e_{3}^{(92)}=(10,0,-1,-1) .
\end{aligned}
$$

Define polytopes $\Delta_{1}, \Delta_{1}^{\prime}, \Delta_{2}, \Delta_{2}^{\prime}, \tilde{\Delta_{3}}, \Delta_{3}$, and $\Delta_{3}^{\prime}$ by

$$
\begin{aligned}
& \Delta_{1}:=\operatorname{Conv}\{(-1,0,0),(0,0,1),(-1,2,0),(-1,3,-1),(2,-1,0)\}, \\
& \Delta_{1}^{\prime}:=\Delta^{(42)}=\operatorname{Conv}\left\{\begin{array}{l}
(-1,-1,1),(-1,-1,-1), \\
(9,-1,-1),(0,2,-1),(-1,2,-1)
\end{array}\right\}, \\
& \Delta_{2}:=\Delta^{(68)}=\operatorname{Conv}\left\{\begin{array}{l}
(-1,0,0),(0,0,1), \\
(-2,4,-1),(-1,3,-1),(2,-1,0)
\end{array}\right\}, \\
& \Delta_{2}^{\prime}:=\operatorname{Conv}\left\{\begin{array}{l}
(-1,-1,1),(-1,-1,-1), \\
(9,-1,-1),(0,2,-1),(-1,1,-1)
\end{array}\right\}, \\
& \tilde{\Delta}_{3}:=\operatorname{Conv}\{(-1,0,0),(1,-1,1),(-1,2,0),(-2,4,-1),(2,-1,0)\} \text {, } \\
& \Delta_{3}:=\Delta^{(83)}=\operatorname{Conv}\left\{\begin{array}{l}
(-1,0,0),(0,0,1), \\
(-2,4,-1),(2,-1,0),(1,-1,1)
\end{array}\right\}, \\
& \Delta_{3}^{\prime}:=\operatorname{Conv}\left\{\begin{array}{l}
(-1,-1,1),(-1,-1,-1), \\
(9,-1,-1),(3,1,-1),(-1,2,-1)
\end{array}\right\} .
\end{aligned}
$$

Since the polar dual polytopes $\Delta_{1}^{*}$ and $\Delta_{2}^{*}$ of $\Delta_{1}$ and $\Delta_{2}$, and $\tilde{\Delta}_{3}^{*}$ and $\Delta_{3}^{*}$ of $\tilde{\Delta}_{3}$ and $\Delta_{3}$ are respectively the convex hulls of vertices $(-1,-1,-1),(1,0,0),(1,3,9)$, $(1,3,-1)$, and $(1,0,-1)$, resp. $(-1,-1,-1),(1,1,3),(1,3,9),(1,3,-1)$, and $(1,0,-1)$, resp. $(-1,-1,-1),(1,0,-1),(1,3,11),(1,3,1)$, and $(1,0,-2)$, resp. $(-1,-1,-1),(1,0,-1),(1,3,11),(1,3,1)$, and $(1,1,-1)$, and the linear maps of $\mathbb{R}^{3}$ determined by matrices $\left(\begin{array}{ccc}0 & 0 & -1 \\ 0 & -1 & -3 \\ -1 & -2 & -5\end{array}\right)$, and $\left(\begin{array}{ccc}0 & 0 & -1 \\ 0 & -1 & -4 \\ -1 & -2 & -6\end{array}\right)$ give isomorphisms from $\Delta_{1}^{\prime}$ to $\Delta_{1}^{*}$ and from $\Delta_{2}^{\prime}$ to $\Delta_{2}^{*}$, respectively from $\Delta_{1}^{\prime}$ to $\tilde{\Delta}_{3}^{*}$ and from $\Delta_{3}^{\prime}$ to $\Delta_{3}^{*}$, the relations $\Delta_{1}^{*} \simeq \Delta_{1}^{\prime}$ and $\Delta_{2}^{*} \simeq \Delta_{2}^{\prime}$, and $\tilde{\Delta}_{3}^{*} \simeq \Delta_{1}^{\prime}$ and $\Delta_{3}^{*} \simeq \Delta_{3}^{\prime}$ hold.

No. 38. The Newton polytope of $F$, which is the convex hull of vertices $(-1,0,0)$, $(0,0,1),(-1,3,-1)$, and $(2,-1,0)$, is not reflexive. Indeed, the polytope $\Delta_{F}$ contains a face spanned by $(-1,0,0),(0,0,1)$, and $(-1,3,-1)$, of which the polar dual is a non-integral vertex $1 / 3(-3,-1,-3)$. Since the complement of the set of lattice points in edges of $\Delta_{F}$ in that of $\Delta^{(68)}$ consists of two lattice points $(-1,2,0)$ and $(-2,4,-1)$, there are only two polytopes possibly to be considered: 
Case 1 The polytope $\Delta$ is spanned by $(-1,0,0),(0,0,1),(-1,2,0),(-1,3,-1)$, and $(2,-1,0)$, namely $\Delta=\Delta_{1}$. In this case, relations $\Delta_{F^{\prime}} \subset \Delta_{1}^{*}=\Delta_{1}^{\prime} \subseteq \Delta^{(42)}$ clearly hold.

Case 2 The polytope $\Delta$ is spanned by $(-1,0,0),(0,0,1),(-2,4,-1),(-1,3,-1)$, and $(2,-1,0)$, namely $\Delta=\Delta_{2}=\Delta^{(68)}$. In this case, relations $\Delta_{F^{\prime}} \subset \Delta_{2}^{*}=\Delta_{2}^{\prime} \subset$ $\Delta^{(42)}$ clearly hold.

Therefore there are two polytope-dual pairs : $\left(\Delta_{1}, \Delta^{(42)}=\Delta_{1}^{\prime}\right)$, and $\left(\Delta^{(68)}=\right.$ $\left.\Delta_{2}, \Delta_{2}^{\prime}\right)$.

No. 39. The Newton polytope of $F$, which is the convex hull of vertices $(-1,0,0)$, $(1,-3,1),(-2,4,-1)$, and $(2,-1,0)$, is easily seen to be reflexive. Besides, the Newton polytope of $F^{\prime}$, which is the convex hull of vertices $(-1,-1,1),(0,-1,-1)$, $(6,0,-1)$, and $(-1,2,-1)$, is not reflexive. It is observed that for any reflexive polytope $\Delta$ such that $\Delta_{F} \subset \Delta \subset \Delta^{(92)}$, the polar dual polytope $\Delta^{*}$ should be a tetrahedron of which each edge contains 3 lattice points. However, there does not exist a reflexive polytope $\Delta^{\prime}$ such that $\Delta_{F^{\prime}} \subset \Delta^{\prime} \subset \Delta^{(42)}$ that contains a vertex which is adjacent to three vertices between which the edges contain 3 lattice points. Thus, in this case, no pair is polytope-dual.

No. 40. The Newton polytope of $F$, which is the convex hull of vertices $(-1,0,0)$, $(1,-1,1),(-2,4,-1)$, and $(2,-1,0)$, is not reflexive. Indeed, the polytope $\Delta_{F}$ contains a face spanned by $(-1,0,0),(1,-1,1)$, and $(-2,4,-1)$, of which the polar dual is a non-integral vertex $1 / 3(3,-1,-7)$. Since the complement of the set of lattice points in edges of $\Delta_{F}$ in that of $\Delta^{(68)}$ consists of two lattice points $(-1,2,0)$ and $(0,0,1)$, there are only two polytopes possibly to be considered:

Case 1 The polytope $\Delta$ is spanned by $(-1,0,0),(1,-1,1),(-1,2,0),(-2,4,-1)$, and $(2,-1,0)$, namely $\Delta=\Delta_{1}$. In this case, relations $\Delta_{F^{\prime}} \subset \Delta_{1}^{*}=\Delta_{1}^{\prime} \subseteq \Delta^{(42)}$ clearly hold.

Case 2 The polytope $\Delta$ is spanned by $(-1,0,0),(0,0,1),(-2,4,-1),(2,-1,0)$, and $(1,-1,1)$, namely $\Delta=\Delta_{3}=\Delta^{(83)}$. In this case, relations $\Delta_{F^{\prime}} \subset \Delta_{3}^{*}=\Delta_{3}^{\prime} \subset$ $\Delta^{(42)}$ clearly hold.

Therefore, ther are two polytope-dual pairs : $\left(\tilde{\Delta}_{1}, \Delta^{(42)}=\Delta_{1}^{\prime}\right)$, and $\left(\Delta^{(83)}=\right.$ $\left.\Delta_{3}, \Delta_{3}^{\prime}\right)$.

\subsection{No. 41-No. 43}

We claim that there exist two polytope-dual pairs for Nos. 41 and 43, and that a unique pair for No. 42. Take a basis $\left\{e_{1}^{(n)}, e_{2}^{(n)}, e_{3}^{(n)}\right\}$ of lattices $M_{n}$ with for $n=25,43,48$, and 88 by

$$
\begin{array}{ll}
e_{1}^{(25)}=(-1,1,0,0), \quad e_{2}^{(25)}=(-3,0,1,0), & e_{3}^{(25)}=(-4,0,0,1), \\
e_{1}^{(43)}=-(1,-8,1,1), e_{2}^{(43)}=(0,-1,2,-1), \quad e_{3}^{(43)}=-(1,1,1,-1), \\
e_{1}^{(48)}=(0,8,-1,-1), e_{2}^{(48)}=-(1,1,-2,1), \quad e_{3}^{(48)}=-(1,1,1,-1), \\
e_{1}^{(88)}=(0,4,-1,-1), e_{2}^{(88)}=(-1,-1,2,-1), e_{3}^{(88)}=(-1,0,-1,1) .
\end{array}
$$


Define polytopes $\Delta_{1}, \Delta_{1}^{\prime}, \Delta_{2}$, and $\Delta_{2}^{\prime}$ by

$$
\begin{aligned}
& \Delta_{1}:=\operatorname{Conv}\{(1,0,0),(0,1,0),(0,0,1),(-2,-6,-9)\}, \\
& \Delta_{1}^{\prime}:=\operatorname{Conv}\{(-1,-1,1),(-1,-1,-1),(8,-1,-1),(-1,2,-1)\}, \\
& \Delta_{2}:=\operatorname{Conv}\{(1,0,0),(0,1,0),(0,0,1),(0,-2,-3),(-1,-3,-4)\}, \\
& \Delta_{2}^{\prime}:=\Delta^{(25)}=\operatorname{Conv}\left\{\begin{array}{l}
(-1,-1,1),(-1,-1,-1), \\
(8,-1,-1),(0,-1,1),(-1,2,-1)
\end{array}\right\} .
\end{aligned}
$$

Since the polar dual polytopes $\Delta_{i}^{*}$ for $i=1,2$ are the convex hulls of vertices $(-1,-1,1),(-1,-1,-1),(8,-1,-1)$, and $(-1,2,-1)$, respectively, $(-1,-1,1)$, $(-1,-1,-1),(8,-1,-1),(0,-1,1)$, and $(-1,2,-1)$, the relations $\Delta_{1}^{*}=\Delta_{1}^{\prime}$ and $\Delta_{2}^{*}=\Delta_{2}^{\prime}$ clearly hold.

Nos. 41 and 43. The Newton polytope of $F$, which is the convex hull of vertices $(1,0,0),(0,1,0),(0,0,1)$, and $(-1,-3,-4)$, is not reflexive. Indeed, the polytope $\Delta_{F}$ contains a face spanned by $(1,0,0),(0,1,0)$, and $(-1,-3,-4)$, of which the polar dual is a non-integral vertex $(-1,-1,5 / 4)$. Since the complement of the set of lattice points on edges of $\Delta_{F}$ in that of $\Delta^{(43)}$ are three points $(1-a,-2 a,-3 a)$ with $a=1,2,3$, we consider a section $\Gamma$ spanned by $(0,1,0),(-1,-3,-4)$, and $(1-a,-2 a,-3 a)$ with $a=1,2,3$. The polar dual of the section $\Gamma$ is a vertex $v=1 /(a-4)(-4(a-1),-(a-4), 2 a-5)$. The vertex $v$ is a lattice point if and only if $a=1,3$. Note that if $a=3$, the polytope is $\Delta_{1}$, and if $a=1$, it is $\Delta_{2}$. The Newton polytope of $F^{\prime}$, which is the convex hull of vertices $(-1,-1,1)$, $(-1,0,-1),(8,-1,-1)$, and $(-1,2,-1)$, is not reflexive. Indeed, the polytope $\Delta_{F^{\prime}}$ contains a face spanned by $(-1,-1,1),(-1,0,-1)$, and $(8,-1,-1)$, of which the polar dual is a non-integral vertex $1 / 11(2,18,9)$. It is clear that both $\Delta_{1}^{\prime}$ and $\Delta_{2}^{\prime}$ are reflexive polytopes that satisfy relations $\Delta_{F^{\prime}} \subset \Delta^{\prime} \subset \Delta^{(25)}$. Therefore, there are two polytope-dual pairs : $\left(\Delta^{(43)}=\Delta_{1}, \Delta_{1}^{\prime}\right)$, and $\left(\Delta_{2}, \Delta_{2}^{\prime}\right)$.

No. 42. The Newton polytope of $F$, which is the convex hull of vertices $(1,0,0)$, $(0,1,0),(0,0,1)$, and $(-1,-3,-4)$, is not reflexive. Indeed, the polytope $\Delta_{F}$ contains a face spanned by $(1,0,0),(0,1,0)$, and $(-1,-3,-4)$ of which the polar dual is a non-integral vertex $(-1,-1,5 / 4)$. Since the complement of the set of lattice points on edges of $\Delta_{F}$ in those of $\Delta^{(88)}$ are three points $(-1,-3,-5),(1-a,-2 a,-3 a)$ with $a=1,2$, consider the following sections:

Case 1 Section spanned by $(-1,-3,-4),(1,0,0)$, and $(-1,-3,-5)$ has the polar dual $(-1,2 / 3,0)$, which is not integral.

Case 2 Section spanned by $(-1,-3,-4),(0,1,0)$, and $(1-a,-2 a,-3 a)$ with $a=1,2$ has the polar dual $v=1 /(a-4)(-4(a-1),-(a-4), 2 a-5)$. The vertex $v$ is a lattice point if and only if $a=1$. Therefore, there are two possibilities: $\Delta^{(88)}$ or $\Delta_{2}$. The polar dual of $\Delta^{(88)}$ contains a vertex $(9,-1,-1)$. However, the polytope $\Delta^{(25)}$ does not contain the vertex. Therefore, $\Delta^{(88)}$ cannot be a polar-dual. It is directly checked that relations $\Delta_{F^{\prime}} \subset \Delta_{2}^{\prime} \subset \Delta^{(25)}$ hold. Therefore, the pair $\left(\Delta_{2}, \Delta_{2}^{\prime}\right)$ is polytope-dual. 


\subsection{No. 44}

We claim that there exists a unique polytope-dual pair. Take a basis $\left\{e_{1}^{(n)}, e_{2}^{(n)}, e_{3}^{(n)}\right\}$ of lattices $M_{n}$ for $n=7$ and 64 by

$$
\begin{gathered}
e_{1}^{(7)}=(-1,1,0,0), \quad e_{2}^{(7)}=(-2,0,1,0), \quad e_{3}^{(7)}=(-4,0,0,1), \\
e_{1}^{(64)}=(-1,5,-1,-1), \quad e_{2}^{(64)}=(0,-1,2,-1), \quad e_{3}^{(64)}=(-1,-1,1,0) .
\end{gathered}
$$

Define polytopes $\Delta_{1}$ and $\Delta_{1}^{\prime}$ by

$$
\begin{aligned}
& \Delta_{1}:=\operatorname{Conv}\{(1,0,0),(0,-1,1),(0,0,1),(-1,2,-6)\} \\
& \Delta_{1}^{\prime}:=\Delta^{(7)}=\operatorname{Conv}\left\{\begin{array}{l}
(-1,-1,1),(-1,-1,-1), \\
(7,-1,-1),(-1,3,-1)
\end{array}\right\} .
\end{aligned}
$$

Since the polar dual polytope $\Delta_{1}^{*}$ is the convex hull of vertices $(-1,2,1),(-1,0,-1)$, $(7,0,-1)$, and $(-1,-4,-1)$, and the linear map of $\mathbb{R}^{3}$ determined by a matrix $\left(\begin{array}{ccc}1 & 0 & 0 \\ 0 & -1 & 0 \\ 0 & 1 & 1\end{array}\right)$ gives an isomorphism from $\Delta_{1}^{\prime}$ to $\Delta_{1}^{*}$, the relation $\Delta_{1}^{*}=\Delta_{1}^{\prime}$ holds.

The Newton polytope of $F$, which is the convex hull of vertices $(1,0,0),(0,-1,1)$, $(0,0,1)$, and $(-1,2,-6)$, coincides with $\Delta_{1}$. Since the complement of the set of lattice points on edges of $\Delta_{F}$ in those of $\Delta^{(64)}$ consists of the only point $(0,1,0)$, the possible $\Delta$ is either $\Delta_{1}$ or $\Delta^{(64)}$. The polar dual of $\Delta^{(64)}$ contains a vertex $(9,-1,-1)$, which does not belong to $\Delta^{(7)}$. Therefore, $\Delta=\Delta_{1}$. It is directly checked that the polar dual $\Delta^{*}=\Delta_{1}^{\prime}$ coincides with $\Delta^{(7)}$. Therefore, the pair $\left(\Delta_{F}=\Delta_{1}, \Delta_{1}^{\prime}\right)$ is polytope-dual.

\subsection{No. 45}

We claim that there exists a unique polytope-dual pair. Take a basis $\left\{e_{1}^{(n)}, e_{2}^{(n)}, e_{3}^{(n)}\right\}$ of lattices $M_{n}$ for $n=35$ and 66 by

$$
\begin{gathered}
e_{1}^{(35)}=(7,0,-1,-1), \quad e_{2}^{(35)}=(6,-1,0,-1), \quad e_{3}^{(35)}=(-1,-1,-1,1), \\
e_{1}^{(66)}=(-1,1,0,0), \quad e_{2}^{(66)}=(-2,0,1,0), \quad e_{3}^{(66)}=(-3,0,0,1) .
\end{gathered}
$$

Define polytopes $\Delta_{1}$ and $\Delta_{1}^{\prime}$ by

$$
\begin{aligned}
& \Delta_{1}:=\operatorname{Conv}\{(0,0,1),(0,1,0),(2,-2,-1),(3,-4,-2),(-2,2,-1)\}, \\
& \Delta_{1}^{\prime}:=\operatorname{Conv}\left\{\begin{array}{l}
(-1,1,0),(0,-1,1), \\
(-1,-1,1),(-1,-1,-1),(6,-1,-1)
\end{array}\right\} .
\end{aligned}
$$

Since the polar dual polytope $\Delta_{1}^{*}$ is the convex hull of vertices $(-1,-1,1)$, $(0,-1,-1),(-2,-1,-1),(-1,0,-1)$, and $(7,6,-1)$, and the linear map of $\mathbb{R}^{3}$ determined by a matrix $\left(\begin{array}{lll}-1 & -1 & 0 \\ -2 & -2 & 1 \\ -4 & -3 & 0\end{array}\right)$ gives an isomorphism from $\Delta_{1}^{\prime}$ to $\Delta_{1}^{*}$, the relation $\Delta_{1}^{*}=\Delta_{1}^{\prime}$ holds. 
The Newton polytope of $F$, which is the convex hull of vertices $(0,0,1),(0,1,0)$, $(3,-4,-2)$, and $(-1,1,0)$, is not reflexive. Indeed, the polytope contains two faces $\Gamma_{1}$ spanned by $(-1,1,0),(0,1,0)$, and $(3,-4,-2)$, of which the polar dual is a nonintegral vertex $(0,-1,2 / 5)$, and $\Gamma_{2}$ spanned by $(0,01),(0,1,0)$, and $(3,-4,-2)$, of which the polar dual is a non-integral vertex $(-7 / 3,-1,-1)$. For $\Gamma_{1}$, one has to make a union of two sections by adding the vertex $(-2,2,-1)$. For $\Gamma_{2}$, consider the section spanned by $(0,1,0),(0,0,1)$, and $(2,-2,-1)$. The polar dual of this section is $(-2,-1,-1)$, which does not belong to $\Delta^{(66)}$. Therefore, the only possibility is $\Delta=\Delta_{1}$. It is directly checked that $\Delta_{F^{\prime}} \subset \Delta_{1}^{\prime} \subset \Delta^{(35)}$. Therefore, the pair $\left(\Delta_{1}, \Delta_{1}^{\prime}\right)$ is polytope-dual.

\subsection{No. 46-No. 47}

We claim that there exists a unique polytope-dual pair for Nos. 46 and 47 . Take a basis $\left\{e_{1}^{(n)}, e_{2}^{(n)}, e_{3}^{(n)}\right\}$ of lattices $M_{n}$ for $n=21,30$ and 86 by

$$
\begin{aligned}
& e_{1}^{(21)}=(-1,1,0,0), \quad e_{2}^{(21)}=(-1,0,1,0), \quad e_{3}^{(21)}=(-2,0,0,1), \\
& e_{1}^{(30)}=(0,4,-1,-1), e_{2}^{(30)}=(-1,-1,4,-1), e_{3}^{(30)}=-(1,1,1,-1), \\
& e_{1}^{(86)}=(4,0,-1,-1), e_{2}^{(86)}=(3,-1,-1,0), \quad e_{3}^{(86)}=(0,-1,2,-1) .
\end{aligned}
$$

Define polytopes $\Delta_{1}, \Delta_{1}^{\prime}, \Delta_{2}$ and $\Delta_{2}^{\prime}$ by

$$
\begin{aligned}
& \Delta_{1}:=\operatorname{Conv}\{(1,0,0),(0,1,0),(0,0,1),(-1,1,0),(2,-3,-1)\}, \\
& \Delta_{1}^{\prime}:=\operatorname{Conv}\left\{\begin{array}{l}
(-1,-1,1),(-1,-1,-1),(4,-1,-1), \\
(-1,4,-1),(-1,2,0),(2,-1,0)
\end{array}\right\}, \\
& \Delta_{2}:=\operatorname{Conv}\{(1,0,0),(0,1,0),(0,0,1),(-2,-2,-5)\}, \\
& \Delta_{2}^{\prime}:=\operatorname{Conv}\{(-1,-1,1),(-1,-1,-1),(4,-1,-1),(-1,4,-1)\} .
\end{aligned}
$$

Since the polar dual polytopes $\Delta_{1}^{*}$ and $\Delta_{2}^{*}$ are the convex hulls of vertices $(0,-1,-1)$, $(-1,-1,-1),(-1,-1,2),(0,-1,4),(5,4,-1),(-1,0,-1)$, res., $(-1,-1,-1)$, $(-1,-1,1),(4,-1,-1),(-1,4,-1)$, and the linear map of $\mathbb{R}^{3}$ determined by a matrix $\left(\begin{array}{lll}-1 & -1 & 1 \\ -1 & -1 & 0 \\ -3 & -2 & 0\end{array}\right)$ gives an isomorphism from $\Delta_{1}^{\prime}$ to $\Delta_{1}^{*}$, the relations $\Delta_{1}^{*} \simeq \Delta_{1}^{\prime}$ and $\Delta_{2}^{*}=\Delta_{2}^{\prime}$ hold.

No. 46. The Newton polytope of $F$, which is the convex hull of vertices $(0,0,1)$, $(0,1,0),(2,-3,-1)$, and $(-1,1,0)$, is not reflexive. Indeed, the polytope $\Delta_{F}$ contains a face spanned by $(0,1,0),(0,0,1)$, and $(2,-3,-1)$, of which the polar dual is $(-5 / 2,-1,-1)$. Since the complement of the set of lattice points on edges of $\Delta_{F}$ in those of $\Delta^{(86)}$ consists of the only $(1,0,0)$, the only possible $\Delta$ is $\Delta^{(86)}=\Delta_{1}$. It is directly checked that relations $\Delta_{F^{\prime}} \subset \Delta_{1}^{\prime}=\Delta^{(86)}$. Therefore, the pair $\left(\Delta^{(86)}=\Delta_{1}, \Delta_{1}^{\prime}\right)$ is polytope-dual.

No. 47. The Newton polytope of $F$, which is the convex hull of vertices $(1,0,0)$, $(0,1,0),(0,0,1)$, and $(-1,-1,-2)$, is not reflexive. Indeed, the polytope $\Delta_{F}$ contains a face spanned by $(1,0,0),(0,1,0)$, and $(-1,-1,-2)$, of which the polar 
dual is $(-1,-1,3 / 2)$. Since the complement of the set of lattice points on edges of $\Delta_{F}$ in those of $\Delta^{(30)}$ consists of the only $(-2,-2,-5)$, the only possible $\Delta$ is $\Delta^{(30)}=\Delta_{2}$. It is directly checked that relations $\Delta_{F^{\prime}} \subset \Delta_{2}^{\prime} \subset \Delta^{(21)}$. Therefore, the pair $\left(\Delta^{(30)}=\Delta_{2}, \Delta_{2}^{\prime}\right)$ is polytope-dual.

\subsection{No. 48-No. 49}

We claim that there exists a unique polytope-dual pair. Take a basis $\left\{e_{1}^{(n)}, e_{2}^{(n)}, e_{3}^{(n)}\right\}$ of lattices $M_{n}$ for $n=5,56$ and 73 by

$$
\begin{gathered}
e_{1}^{(5)}=(-1,1,0,0), \quad e_{2}^{(5)}=(-1,0,1,0), \quad e_{3}^{(5)}=(-3,0,0,1), \\
e_{1}^{(56)}=(-1,0,2,-1), \quad e_{2}^{(56)}=(-1,-1,0,1), \quad e_{3}^{(56)}=(5,-1,-1,-1), \\
e_{1}^{(73)}=(-1,-1,4,-1), \quad e_{2}^{(73)}=(-1,-1,-1,1), \quad e_{3}^{(73)}=(5,0,-1,-1) .
\end{gathered}
$$

In Kobayashi and Mase (2012), it is proved that the polytopes $\Delta^{(56)}$ and $\Delta^{(73)}$ are isomorphic to the polytope $\Delta_{(56,73)}$ with vertices $(1,0,0),(0,1,0),(0,0,1)$, and $(-1,-3,-1)$, under the above choice of basis. Let $\Delta_{1}$ be $\Delta_{(56,73)}$, and $\Delta_{1}^{\prime}$ be the polytope $\Delta^{(5)}$ which is the convex hull of vertices $(-1,-1,1),(-1,-1,-1)$, $(5,-1,-1)$, and $(-1,5,-1)$. Since the polar dual polytope $\Delta_{1}^{*}$ of $\Delta_{1}$ is the convex hull of vertices $(-1,-1,-1),(-1,1,-1),(5,-1,-1)$, and $(-1,-1,5)$, and the linear map of $\mathbb{R}^{3}$ determined by a matrix $\left(\begin{array}{lll}1 & 0 & 0 \\ 0 & 0 & 1 \\ 0 & 1 & 0\end{array}\right)$ gives an isomorphism from $\Delta_{1}^{\prime}$ to $\Delta_{1}^{*}$, the relation $\Delta_{1}^{*} \simeq \Delta_{1}^{\prime}$ holds.

Nos. 48 and 49. In both cases, the Newton polytope of $F$, which is the convex hull of vertices $(1,0,0),(0,1,0),(0,0,1)$, and $(-1,-3,-1)$, coincides with $\Delta_{1}$. It is directly checked that the Newton polytope of $F^{\prime}$ is a subpolytope of $\Delta_{1}^{\prime}$. Therefore, the pair $\left(\Delta_{F}=\Delta_{1}, \Delta_{1}^{\prime}\right)$ is the only polytope-dual.

\subsection{No. 50}

We claim that there exists a unique polytope-dual pair. Take a basis $\left\{e_{1}^{(n)}, e_{2}^{(n)}, e_{3}^{(n)}\right\}$ of lattices $M_{n}$ for $n=1$ and 52 by

$$
\begin{gathered}
e_{1}^{(1)}=(-1,1,0,0), \quad e_{2}^{(1)}=(-1,0,1,0), \quad e_{3}^{(1)}=(-1,0,0,1), \\
e_{1}^{(52)}=(-1,2,-1,0), \quad e_{2}^{(52)}=(-1,-1,3,-1), \quad e_{3}^{(52)}=(-1,-1,-1,2) .
\end{gathered}
$$

Define polytopes $\Delta_{1}$ and $\Delta_{1}^{\prime}$ by

$$
\begin{aligned}
& \Delta_{1}:=\Delta^{(52)}=\operatorname{Conv}\{(1,0,0),(0,1,0),(0,0,1),(-1,-1,-1)\}, \\
& \Delta_{1}^{\prime}:=\Delta^{(1)}=\operatorname{Conv}\{(-1,-1,3),(-1,-1,-1),(3,-1,-1),(-1,3,-1)\} .
\end{aligned}
$$

Since the polar dual polytope $\Delta_{1}^{*}$ of $\Delta_{1}$ is the convex hull of vertices $(-1,-1,3)$, $(-1,-1,-1),(3,-1,-1)$, and $(-1,3,-1)$, the relation $\Delta_{1}^{*}=\Delta_{1}^{\prime}$ clearly holds. 
The Newton polytope of $F$, which is the convex hull of vertices $(1,0,0),(0,1,0)$, $(0,0,1)$, and $(-1,-1,-1)$, coincides with $\Delta_{1}$. Thus, $\Delta=\Delta_{F}=\Delta^{(52)}=\Delta_{1}$ is the only possible reflexive polytope. The polar dual $\Delta_{1}^{\prime}$ coincides with $\Delta^{(1)}$ and thus the Newton polytope $\Delta_{F^{\prime}}$ of $F^{\prime}$ is a subpolytope of $\Delta_{1}^{\prime}$. Therefore, the pair $\left(\Delta_{F}=\Delta_{1}, \Delta^{(1)}=\Delta_{1}^{\prime}\right)$ is polytope-dual.

\subsection{No. 51}

We claim that there exist sixteen polytope-dual pairs. Take a basis $\left\{e_{1}^{(n)}, e_{2}^{(n)}, e_{3}^{(n)}\right\}$ of the lattice $M_{32}$ by

$$
e_{1}^{(32)}=(-1,0,3,-1), \quad e_{2}^{(32)}=(0,-1,3,-1), \quad e_{3}^{(32)}=(-1,-1,-1,1) .
$$

Define polytopes $\Delta_{i, a}$ and $\Delta_{i^{\prime}, a}$ with $i=1,2$ by

$$
\begin{aligned}
& \Delta_{1, a}:=\operatorname{Conv}\left\{\begin{array}{l}
(0,0,1),(1,0,0),(0,1,0),(-4,3,-2),\}, \\
(-a+3, a-4,-2),(2,-2,-1)
\end{array}\right\}, \\
& \Delta_{1^{\prime}, a}:=\operatorname{Conv}\left\{\begin{array}{l}
(0,0,1),(2,-2,-1),(0,1,0),(-2,2,-1), \\
(-a+3, a-4,-2),(3,-4,-2)
\end{array}\right\}, \\
& \Delta_{2, a}:=\operatorname{Conv}\left\{\begin{array}{l}
(0,0,1),(1,0,0),(-2,2,-1),(-4,3,-2), \\
(-a+3, a-4,-2),(2,-2,-1)
\end{array}\right\}, \\
& \Delta_{2^{\prime}, a}:=\operatorname{Conv}\left\{\begin{array}{l}
(0,0,1),(1,0,0),(0,1,0),(-2,2,-1), \\
(-a+3, a-4,-2),(3,-4,-2)
\end{array}\right\},
\end{aligned}
$$

where $a=0,1, \ldots, 7$.

Since the polar dual polytopes $\Delta_{1, a}^{*}$ and $\Delta_{2, a}^{*}$ of $\Delta_{1, a}$ and $\Delta_{2, a}$ are respectively the convex hulls of vertices $(-1,-1,1),(-1,-1,-1),(0,-1,-1),(3,3,-1),(a-$ $1, a,-1)$, and $(-1,0,-1)$; and $(-1,-1,1),(-1,-2,-1),(0,-1,-1),(3,3,-1)$, $(a-1, a,-1)$ and $(-1,0,-1)$, and the linear map of $\mathbb{R}^{3}$ determined by a matrix $\left(\begin{array}{ccc}-1 & -2 & -1 \\ 0 & -1 & -1 \\ -1 & -1 & 1\end{array}\right)$ gives an isomorphism from $\Delta_{i, a}^{\prime}$ to $\Delta_{i, a}^{*}$, the relation $\Delta_{i, a}^{*} \simeq \Delta_{i, a}^{\prime}$ for $i=1,2$ holds.

The Newton polytope of $F=F^{\prime}$, which is the convex hull of vertices $(1,0,0)$, $(-4,3,-2),(0,0,1)$, and $(2,-2,-1)$, is not reflexive. Indeed, the polytope $\Delta_{F}$ contains a face spanned by $(1,0,0),(0,0,1)$, and $(-4,3,-2)$, of which the polar dual is a non-integral vertex $(-1,-7 / 3,-1)$.

Since the complement of the set of lattice points on edges of $\Delta_{F}$ in those of $\Delta^{(32)}$ consists of $(2,-2,-1),(1,0,0)$, and $(-a+3, a-4,-2)$ with $a=0,1, \ldots, 7$, it is clear that we just need to consider polytopes of form $\Delta_{1, a}$ and $\Delta_{2, a}$ with $a=$ $0,1, \ldots, 7$. And for each $a$, we can find a polytope-dual partner.

Therefore, the claims are verified. 


\section{Closing Remarks}

As is mentioned in Ebeling (2006), almost all mirror symmetric pairs of weight systems in the sense of Belcastro (2002) are also strongly coupled. We would like to study not only the full families of $K 3$ surfaces, but families of $K 3$ surfaces associated to reflexive polytopes $\Delta$ and $\Delta^{\prime}$ obtained in Theorem 5.1. More precisely, it is interesting to study a relation with a lattice duality (Dolgachev 1996) and the coupling.

Acknowledgements Open Access funding provided by Projekt DEAL. The author thanks to Professor Wolfgang Ebeling for his suggestion of the study and discussions.

Open Access This article is licensed under a Creative Commons Attribution 4.0 International License, which permits use, sharing, adaptation, distribution and reproduction in any medium or format, as long as you give appropriate credit to the original author(s) and the source, provide a link to the Creative Commons licence, and indicate if changes were made. The images or other third party material in this article are included in the article's Creative Commons licence, unless indicated otherwise in a credit line to the material. If material is not included in the article's Creative Commons licence and your intended use is not permitted by statutory regulation or exceeds the permitted use, you will need to obtain permission directly from the copyright holder. To view a copy of this licence, visit http://creativecommons.org/licenses/by/4.0/.

\section{References}

Batyrev, V.V.: Dual polyhedra and mirror symmetry for Calabi-Yau hypersurfaces in toric varieties. J. Algebr. Geom. 3, 493-535 (1994)

Belcastro, S.-M.: Picard lattices of families of $K 3$ surfaces. Commun. Algebra 30, 61-82 (2002)

Dolgachev, I.: Weighted projective varieties. In: Group Actions and Vector Fields, LNM, vol. 956. Springer, pp. 34-72 (1982)

Dolgachev, I.: Mirror symmetry for lattice polarized K3 surfaces. J. Math. Sci. 81, 2599-2630 (1996)

Ebeling, W.: Mirror symmetry, Kobayashi's duality, and Saito's duality. Kodai Math. J. 29, 319-336 (2006)

Ebeling, W., Takahashi, A.: Strange duality of weighted homogeneous polynomials. Compos. Math. 147(5), 1413-1433 (2011)

Kobayashi, M.: Duality of weights, mirror symmetry and Arnold's strange duality. Tokyo J. Math. 31, 225-251 (2008)

Kobayashi, M., Mase, M.: Isomorphism among families of weighted $K 3$ hypersurfaces. Tokyo J. Math. 35, 461-468 (2012)

Mase, M., Ueda, K.: A note on bimodal singularities and mirror symmetry. Manuscr. Math. 146, 153-177 (2015) (online 31 August 2014)

Yonemura, T.: Hypersurface simple $K 3$ singularities. Tôhoku Math. J. 42, 351-380 (1990)

Publisher's Note Springer Nature remains neutral with regard to jurisdictional claims in published maps and institutional affiliations. 